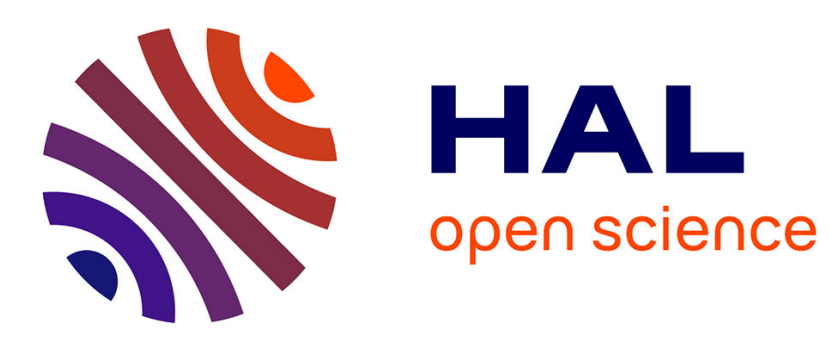

\title{
A Nonlinear Quantum Adiabatic Approximation
}

Clotilde Fermanian Kammerer, Alain Joye

\section{To cite this version:}

Clotilde Fermanian Kammerer, Alain Joye. A Nonlinear Quantum Adiabatic Approximation. Nonlinearity, 2020, 33, pp.4715-4751. hal-02163163v2

\section{HAL Id: hal-02163163 \\ https://hal.science/hal-02163163v2}

Submitted on 12 Oct 2020

HAL is a multi-disciplinary open access archive for the deposit and dissemination of scientific research documents, whether they are published or not. The documents may come from teaching and research institutions in France or abroad, or from public or private research centers.
L'archive ouverte pluridisciplinaire HAL, est destinée au dépôt et à la diffusion de documents scientifiques de niveau recherche, publiés ou non, émanant des établissements d'enseignement et de recherche français ou étrangers, des laboratoires publics ou privés. 


\title{
A Nonlinear Quantum Adiabatic Approximation
}

\author{
Clotilde Fermanian-Kammerer * $\quad$ Alain Joye $^{\dagger}$
}

\begin{abstract}
This paper is devoted to a generalisation of the quantum adiabatic theorem to a nonlinear setting. We consider a Hamiltonian operator which depends on the time variable and on a finite number of parameters and acts on a separable Hilbert space of which we select a fixed basis. We study an evolution equation in which this Hamiltonian acts on the unknown vector, while depending on coordinates of the unknown vector in the selected basis, thus making the equation nonlinear. We prove existence of solutions to this equation and consider their asymptotics in the adiabatic regime, i.e. when the Hamiltonian is slowly varying in time. Under natural spectral hypotheses, we prove the existence of normalised time dependent vectors depending on the Hamiltonian only, called instantaneous nonlinear eigenvectors, and show the existence of solutions which remain close to these vectors, up to a rapidly oscillating phase, in the adiabatic regime. We first investigate the case of bounded operators and then exhibit a set of spectral assumptions under which the result extends to unbounded Hamiltonians.
\end{abstract}

Keywords: Adiabatic approximation, nonlinear adiabatic theorem.

Acknowledgments: This work is partially supported by the ANR grant NONSTOPS (ANR-17-CE400006-01), by the Von Neumann visiting professorship of the Technische Universität München and the CNRS Project 80|Prime AlgDynQua. It has been partially written in the Mathematics Department of the Technische Universität München and during a visit of AJ at the Institut Mittag-Leffler. The authors thank these two institutions for their warm hospitality, with a special thought for the stimulating discussions with Simone Warzel and Herbert Spohn.

\section{Introduction}

We consider a time dependent Hamiltonian on a separable Hilbert space $\mathcal{H}$ that depends on a finite number of real parameters taken in some open neighborhoods $\mathcal{T}$ and $\mathcal{X}$ of $[0,1]$ :

$$
\mathcal{T} \times \mathcal{X}^{p} \ni(t, x) \rightarrow H(t, x) \in \mathcal{L}(\mathcal{H})
$$

where $H(t, x)$ is a smooth map, i.e. $\mathcal{C}^{\infty}$, valued in the set of self-adjoint operators on $\mathcal{H}$. Let $\left\{e_{j}\right\}_{j \in \mathbb{N}}$ be a fixed orthonormal basis of $\mathcal{H}$ and for $f \in \mathcal{H}$, we denote by $f_{j}$ its coordinate along $e_{j}$, i.e. $f_{j}=\left\langle e_{j} \mid f\right\rangle$. We consider solutions to the nonlinear evolution equation

$$
i \varepsilon \partial_{t} v^{\varepsilon}(t)=H\left(t,\left|v_{1}^{\varepsilon}(t)\right|^{2}, \ldots,\left|v_{p}^{\varepsilon}(t)\right|^{2}\right) v^{\varepsilon}(t), \quad v^{\varepsilon}(0)=v_{0},
$$

for $t \in \mathcal{T}$ and initial data $v_{0} \in \mathcal{H}$ with $\left\|v_{0}\right\|=1$, in the limit where the small parameter $\varepsilon$ tends to zero.

*Université Paris Est Créteil, Université Gustave Eiffel, CNRS, LAMA, UMR CNRS 8050, 61, avenue du Général de Gaulle, 94010 Créteil Cedex, France,

${ }^{\dagger}$ Univ. Grenoble Alpes, CNRS, Institut Fourier, F-38000 Grenoble, France 
More precisely, we prove under natural spectral hypotheses that for the systems we consider, there exist an interval of times $\mathcal{T}_{0} \subset \mathcal{T}$ (containing 0 ) and a family of smooth nonlinear eigenvectors, i.e. two smooth maps $t \mapsto \omega(t) \in \mathcal{H}$ and $t \mapsto \lambda(t) \in \mathbb{R}$, such that $\|\omega(t)\| \equiv 1,\left\langle\omega(t) \mid \partial_{t} \omega(t)\right\rangle \equiv 0$, and

$$
H\left(t,\left|\omega_{1}(t)\right|^{2}, \ldots,\left|\omega_{p}(t)\right|^{2}\right) \omega(t)=\lambda(t) \omega(t), \quad \forall t \in \mathcal{T}_{0}
$$

and we provide conditions under which the deviations of $v^{\varepsilon}(t)$ from $\omega(t) \mathrm{e}^{-\frac{i}{\varepsilon} \int_{0}^{t} \lambda(s) d s}$ are small as $\varepsilon \rightarrow 0$, in the case where the initial data is taken along $\omega(0)\left(v^{\varepsilon}(0)=\omega(0)\right)$.

We stress that the evolution equation (1.2) depends on the choice of the first $p$ vectors of the orthonormal basis $\left\{e_{j}\right\}_{j \in \mathbb{N}}$. It is also important to note that the norm of $v^{\varepsilon}(t)$ is preserved and, because of the choice of a normalized initial data, $\left\|v^{\varepsilon}(t)\right\|$. In particular, this implies

$$
\left|v_{j}^{\varepsilon}(t)\right|^{2} \in[0,1] \subset \mathcal{X}, \forall j \in\{1, \cdots p\} .
$$

The limit $\varepsilon \rightarrow 0$ that we consider is known as the adiabatic limit and consists in analyzing in finite time the evolution of slowly varying Hamiltonian: indeed, with the change of variable $t=\varepsilon s$ and of unknown function $\tilde{v}^{\varepsilon}(s)=v^{\varepsilon}(t)$, equation 1.2 is equivalent to

$$
i \partial_{s} \tilde{v}^{\varepsilon}=H\left(\varepsilon s,\left|\tilde{v}_{1}^{\varepsilon}(s)\right|^{2}, \ldots,\left|\tilde{v}_{p}^{\varepsilon}(s)\right|^{2}\right) \tilde{v}^{\varepsilon}(s), \quad \tilde{v}^{\varepsilon}(0)=v_{0}
$$

where the map $s \mapsto H(\varepsilon s, x)$ is slowly varying in $s \in \mathcal{T} / \varepsilon$. In the context of linear equations, such an analysis leads to the celebrated adiabatic theorem of quantum mechanics see e.g. [K1]. Our aim here is to provide a framework where one can prove an approximation of the solution to the nonlinear equation (1.2) that bears some similarities with the well-known adiabatic results of the litterature.

The adiabatic theorem of quantum mechanics has found numerous extensions since its first formulations [BF, K1] for self-adjoint time dependent Hamiltonians with an isolated eigenvalue. It was extended to accommodate isolated parts of spectrum [N1, ASY] and it was shown to be exponentially accurate for analytic time dependence [JKP, JP, N2, J1]. Then, it was extended to deal with gapless situations where the eigenvalue of interest is not isolated in the rest of the spectrum, $\mathrm{AHS}$, $\mathrm{AE}, \mathrm{Te}$. Generalisations to non-self-adjoint generators were provided in [A-SF, J3, AFGG], leading to extensions to gapless, non self-adjoint generators provided in $[\mathrm{Sc}$. Also, formulations of the adiabatic approximation have been shown to hold true for unitary and non unitary discrete time evolutions, [DKS, Ta, HJPR1, HJPR2, and for extended many body systems [BDR. From this perspective, we prove a generalisation of the adiabatic theorem to nonlinear non-autonomous evolution equations in a Hilbert space defined by $(1.2)$ and (1.1).

Such nonlinear evolution equations occur for example in condensed matter Physics or nonlinear Optics within certain parameter regimes. In particular, the analysis of Landau-Zener tuneling of a Bose-Einstein condensate between Bloch bands in an optical lattice or in double well potentials, as in $[\mathrm{BQ}$, [J-L et al., $\mathrm{Kh}, \mathrm{KhRu}]$ or the study of optical waveguides known as nonlinear coherent couplers $[\mathrm{Je}, \mathrm{A}$, lead to systems of this form. Indeed, within a certain regime, the relevant Hamiltonians take the explicit form (1.2) for $p=2$ with an explicit two by two matrix $H\left(t, x_{1}, x_{2}\right)$, see the book [LLFY] for examples and more references. A concrete example is provided by the work [MCWW] in which the dynamics of a Bose-Einstein condensate in a double-well potential are studied, within the meanfield and two-mode approximations. In this regime, one considers the Gross-Pitaevskii equation for 
the condensate, assuming the two wells of the potential are sufficiently deep and separated so that the condensate wave function can be expressed as a linear combination of the ground states in each of the two wells. Under suitable assumptions on the many-body interaction term, the resulting effective evolution of the coefficients $\left(v_{1}, v_{2}\right) \in \mathbb{C}^{2}$ of this expansion that describe the number of particles in each well, takes the form

$$
i \partial_{t}\left(\begin{array}{l}
v_{1} \\
v_{2}
\end{array}\right)=\left(\begin{array}{cc}
\kappa\left|v_{1}\right|^{2} & \Omega \\
\Omega & \kappa\left|v_{2}\right|^{2}
\end{array}\right)\left(\begin{array}{l}
v_{1} \\
v_{2}
\end{array}\right)
$$

where $\Omega$ and $\kappa$ are parameters which depend on time when the two-well potential depends on time. This yields a Hamiltonian of the type (1.1). While the quadratic dependence in the components $\left|v_{j}\right|$ of the Hamiltonian above is dictated by the Physics it describes, our results hold for Hamiltonians displaying arbitrary smooth dependence in $\left|v_{j}\right|^{2}$.

Adiabatic issues have been already addressed in the PDE literature in a nonlinear setting with different perspectives. With a scattering point of view, the long time behaviour of nonlinear two by two problems with generators similar to those mentioned above was analysed by [CFK2]. In a PDE setting, [CFK1 and [ $\mathrm{H}]$ study the adiabatic propagation of coherent states for systems of Schrödinger equations with a non linearity and [S] considers the adiabatic regime of the nonlinear Schrödinger equation for small data. A common feature of these works is that the effective nonlinearity is weak in the sense that it decays with $\varepsilon$. This is not the case in GG which studies a PDE with a nonlinearity of order $\mathcal{O}(1)$ as $\varepsilon \rightarrow 0$, for small initial data, but of size independent of $\varepsilon$. The authors consider therein the time dependent Gross-Pitaevskii equation in a potential which varies slowly in time. Under suitable conditions on the potential, a unique ground state exists for the stationary linear equation parametrized by the time variable, playing the role of a nonlinear eigenvector in the sense of the previous paragraphs, and the solution to the Gross-Pitaevskii equation is shown to follow the instantaneous ground state, for large times.

Our approach here is closer to the latter reference. Indeed, we aim at providing a general functional framework for nonlinear adiabatic evolution equations (1.2) and (1.1), characterised by non linearities of order $\mathcal{O}(1)$ as $\varepsilon \rightarrow 0$ and admitting solutions of norm strictly equal to one, in contrast to the PDE results mentionned above. We then discuss a set of reasonable spectral hypotheses on $H(t, x)$ allowing us to provide an approximation of the solutions to (1.2) as $\varepsilon \rightarrow 0$, for times $t$ of order one. Our main result is first proven for bounded Hamiltonians, and then extended to unbounded $H(t, x)$, under suitable spectral assumptions. In particular, the latter case applies to a certain type of nonlinear Schrödinger equation on $L^{2}(\mathbb{R})$ that we discuss.

Note that the matrix cases considered in CFK2 or [LLFY] and in the references therein, appear as special cases of those that we consider, whereas our hypotheses excludes the PDE setup considered in [CFK1, H, S, GG]. This is due to the fact that the nonlinearity in 1.2 depends on the norm of the projections of the wave function on some subset of the basis vectors of the Hilbert space, and not of the

modulus of the wave function itself as in the Gross-Pitaievski equation or in Hartree equation. In this sense, the nonlinearity that we consider is weaker.

\subsection{Setup and main result}

To ease notations, we will write from now on

$$
H\left(t,\left|v_{1}\right|^{2}, \ldots,\left|v_{p}\right|^{2}\right)=: H(t,[v]),
$$


for any vector $v \in \mathcal{H}$, where $H$ depends on $p<\infty$ components of $v$ only. The form of the nonlinearity we choose, depending on the modulus of (certain components of) the solution, is reminiscent of that of the nonlinear Schrödinger equation. It entails in particular the fact that $H$ actually depends on $\left\{v_{1}, \bar{v}_{1}, v_{2}, \bar{v}_{2}, \ldots v_{p}, \bar{v}_{p}\right\}$. This motivates the introduction of the anti-unitary complex conjugation $C$ on $\mathcal{H}$ defined by

$$
\forall v=\sum_{j} v_{j} e_{j} \in \mathcal{H}, \quad C v=\sum_{j} \bar{v}_{j} e_{j}
$$

to be used later on. Note that $C$ depends on the basis $\left\{e_{j}\right\}_{j \in \mathbb{N}}$ that is considered invariant under $C$. For any $A \in \mathcal{L}(\mathcal{H})$, we define the operator $\bar{A}=C A C \in \mathcal{L}(\mathcal{H})$ and will call operators such that $\bar{A}=A$, real operators. We will work under the following general hypotheses.

$\mathbf{H}_{0}$ The map $\mathcal{T} \times \mathcal{X}^{p} \ni(t, x) \mapsto H(t, x) \in \mathcal{L}(\mathcal{H})$ is $C^{\infty}$ in the operator norm topology, where $\mathcal{T}$ and $\mathcal{X}$ are open neighbourhoods of $[0,1]$. For all $(t, x) \in \mathcal{T} \times \mathcal{X}^{p}, H(t, x)=H^{*}(t, x)$.

$\mathbf{H}_{1}$ There exists $\delta>0$ such that $\left\|\partial_{x_{j}} H(x, t)\right\| \leq \delta$, for all $(t, x) \in \mathcal{T} \times \mathcal{X}^{p}$ and $j \in\{1, \ldots, p\}$.

$\mathbf{H}_{2}$ For all $(t, x) \in \mathcal{T} \times \mathcal{X}^{p}$, the spectrum $\sigma(H(x, t))$ consists in $N$ distinct eigenvalues $\left\{\lambda_{j}(t, x)\right\}_{j=1}^{N}$, possibly degenerate, that are separated from one another by a gap bounded below by $g>0$, uniformly in $(t, x)$.

$\mathbf{H}_{3}$ There exists $1 \leq j_{0} \leq N$ such that $\lambda_{j_{0}}(x, t)$ is simple.

Consequently, the corresponding spectral decomposition of $H(t, x)$ reads

$$
H(t, x)=\sum_{j=1}^{N} \lambda_{j}(t, x) P_{j}(t, x),
$$

where the orthogonal spectral projectors $P_{j}(t, x)$ have constant rank which may be infinite, while $\operatorname{dim}\left(P_{j_{0}}(t, x)\right) \equiv 1$. We shall make use of the following facts: the maps $(t, x) \mapsto P_{j}(t, x)$ are $\mathcal{C}^{\infty}$ and so are $(t, x) \mapsto \lambda_{j}(t, x)$. Moreover, for $j=j_{0}$, there exists a global smooth map $\mathcal{T} \times \mathcal{X}^{p} \ni(t, x) \mapsto \varphi_{j_{0}}(t, x) \in \mathcal{H}$ such that $\left\|\varphi_{j_{0}}(t, x)\right\| \equiv 1$ and

$$
\forall(t, x) \in \mathcal{T} \times \mathcal{X}^{d}, \quad H(t, x) \varphi_{j_{0}}(t, x)=\lambda_{j_{0}}(t, x) \varphi_{j_{0}}(t, x) .
$$

These facts are briefly discussed in Section 2 below.

The form of the nonlinearity immediately implies a gauge invariance, which will turn out to be crucial later on. Due to 1.3 , we have for any $\theta \in \mathbb{R}$, any $v \in \mathcal{H}$,

$$
H\left(t,\left[\mathrm{e}^{i \theta} v\right]\right)=H(t,[v]) .
$$

If $\mathbf{H}_{2}$ and $\mathbf{H}_{3}$ hold as well, this implies

$$
P_{j}\left(t,\left[\mathrm{e}^{i \theta} v\right]\right)=P_{j}(t,[v]), \quad \lambda_{j}\left(t,\left[\mathrm{e}^{i \theta} v\right]\right)=\lambda_{j}(t,[v]), \quad \varphi_{j_{0}}\left(t,\left[\mathrm{e}^{i \theta} v\right]\right)=\varphi_{j_{0}}(t,[v]) .
$$

We first note that the self-adjointness of $H(t, x)$ ensures that $\left\|v^{\varepsilon}(t)\right\|=\left\|v_{0}\right\|=1$, whence the existence of global solutions to (1.2) via Cauchy-Lipschitz Theorem. Moreover, gauge invariance (1.6) implies symmetries that we exploit below. These elementary properties are stated in the next Lemma with the convention 1.3 . 
Lemma 1.1 Under assumption $\mathbf{H}_{0}$, for any $v_{0} \in \mathcal{H}$, the equation

$$
i \varepsilon \partial_{t} v^{\varepsilon}(t)=H\left(t,\left[v^{\varepsilon}(t)\right]\right) v^{\varepsilon}(t), \quad v^{\varepsilon}(0)=v_{0} \in \mathcal{H}, t \in \mathcal{T},
$$

admits a unique global solution with $\left\|v^{\varepsilon}(t)\right\|=\left\|v_{0}\right\|$.

Besides, given a $C^{0}$ map $\mathcal{T} \times \mathcal{X}^{p} \ni(t, x) \rightarrow \chi(t, x) \in \mathbb{R}$, and $v^{\varepsilon}(t)$ a solution to (1.8), the solution to

$$
i \varepsilon \partial_{t} s^{\varepsilon}(t)=\left(H\left(t,\left[s^{\varepsilon}(t)\right]\right)+\chi\left(t,\left[s^{\varepsilon}(t)\right]\right) \mathrm{Id}\right) s^{\varepsilon}(t), \quad s^{\varepsilon}(0)=v_{0} \in \mathcal{H}, t \in \mathcal{T}
$$

reads

$$
s^{\varepsilon}(t)=\mathrm{e}^{-i \int_{0}^{t} \chi\left(u,\left[v^{\varepsilon}(u)\right]\right) d u / \varepsilon} v^{\varepsilon}(t), \quad \forall t \in \mathcal{T} .
$$

Our analysis focuses on solutions to 1.2 that are tightly related to the simple eigenvalue $\lambda_{j_{0}}(t, x)$ and associated eigenvector $\varphi_{j_{0}}(t, x)$. Therefore, to simplify the notation, we drop the index $j_{0}$ for these spectral data from now on. We start by introducing a vector $\omega(t) \in \mathcal{H}$ defined in a neighbourhood of $0 \in \mathcal{T}_{0}$ by

$$
H(t,[\omega(t)]) \omega(t)=\lambda(t,[\omega(t)]) \omega(t), \quad \forall t \in \mathcal{T}_{0} .
$$

As discussed in Section 2, this nonlinear equation (that does not involve any derivative of $\omega(t)$ ) turns out to always have a local nontrivial solution when $\lambda(t, x)$ is a simple eigenvalue of $H(t, x)$.

Proposition 1.2 Assume $\mathbf{H}_{0}, \mathbf{H}_{1}, \mathbf{H}_{2}$ and $\mathbf{H}_{3}$. Then, for any $t_{0} \in \mathcal{T}$, there exists a neighbourhood $\mathcal{T}_{0} \subset \mathcal{T}$ of $t_{0}$ such that for all $t \in \mathcal{T}_{0}$, there exists a solution $\omega(t) \in \mathcal{H}$ of norm one to the equation

$$
P(t,[\omega(t)]) \omega(t)=\omega(t) .
$$

Moreover, there exists $\delta_{0}>0$ such that for $\delta \in\left(0, \delta_{0}\right)$, the map $\mathcal{T}_{0} \ni t \mapsto \omega(t)$ is $\mathcal{C}^{\infty}$ and can be chosen to satisfy

$$
\left\langle\omega(t) \mid \partial_{t} \omega(t)\right\rangle \equiv 0,
$$

which makes it unique up to a constant phase.

In the sequel, we shall always make the choice 1.10 and we will call such a vector an instantaneous nonlinear eigenvector. We can now give our main statements which establish nonlinear adiabatic theorems in the considered framework. We first consider the case $\mathcal{H}=\mathbb{C}^{N}$.

Theorem 1.3 Assume $\mathcal{H}=\mathbb{C}^{N}, \mathbf{H}_{0}, \mathbf{H}_{1}$ with $\delta$ small enough, and suppose that $\mathbf{H}_{2}$ holds with all eigenvalues being simple. Moreover, assume that $H(t, x)$ is real, that is $\bar{H}(t, x)=H(t, x)$, and generic in the sense that $\sigma(H(t, x)-\lambda(t, x)) \cap \sigma(-H(t, x)+\lambda(t, x))=\{0\}$. Let $\omega(t)$ be the instantaneous nonlinear eigenvector defined in Proposition 1.2 in a neighbourhood $\mathcal{T}_{0}$ of $t_{0}=0$. Then the solution $v^{\varepsilon}(t)$ to 1 .2) with $v^{\varepsilon}(0)=\omega(0)$ satisfies for all $t \in \mathcal{T}_{0}$

$$
v^{\varepsilon}(t)=\mathrm{e}^{-\frac{i}{\varepsilon} \int_{0}^{t} \lambda(s,[\omega(s)]) d s} \omega(t)+O_{t}(\varepsilon) .
$$

Remark 1.4 i) Note that the condition on the smallness of $\delta$ is independent on $\varepsilon$.

ii) The genericity condition always holds if $\lambda(t, x)$ is the ground state or the largest eigenvalue of $H(t, x)$. 
After a reduction to the case where $\lambda(t, x)=0$, the proof of this theorem relies on the analysis of the system satisfied by the element $(\Delta(t), \bar{\Delta}(t))$ of $\mathcal{H} \times \mathcal{H}$ where

$$
\Delta(t)=v^{\varepsilon}(t)-\omega(t) .
$$

Setting ${ }^{\cdot}=\partial_{t}$, a linearization process around $\omega(t)$ shows that the evolution of $\Delta(t)$ is driven by an evolution equation of the form

$$
i \varepsilon\left(\begin{array}{c}
\dot{\Delta} \\
\dot{\bar{\Delta}}
\end{array}\right)=F(t)\left(\frac{\Delta}{\bar{\Delta}}\right)-i \varepsilon\left(\begin{array}{c}
\dot{\omega} \\
\dot{\bar{\omega}}
\end{array}\right)+\left(\begin{array}{c}
r^{\varepsilon} \\
-\bar{r}^{\varepsilon}
\end{array}\right), \Delta(0)=0,
$$

with $r^{\varepsilon}(t)=O\left(\|\Delta(t)\|^{2}\right)$ and $F(t)=F_{0}(t)+G(t)$ for some finite rank non self-adjoint operator $G(t)$ and

$$
F_{0}(t)=\left(\begin{array}{cc}
H(t,[\omega(t)]) & 0 \\
0 & -\bar{H}(t,[\omega(t)])
\end{array}\right) .
$$

The smallness of $\Delta(t)$ is then proved thanks to a careful analysis of this equation in which the spectrum of $F(t)$ plays a crucial role. The conditions on the spectrum of $H(t, x)-\lambda(t, x)$ that are assumed in Theorem (1.3) allow to ensure that the operator $F(t)$ is semisimple with real eigenvalues of constant multiplicity for all $t \in \mathcal{T}_{0}$, which is enough to develop an approach $\grave{a}$ la Kato and prove that there exist positive constants $c_{0}, c_{1}$ such that the norm of the remainder satisfies

$$
\|\Delta(t)\| \leq \min \left(c_{0} t, c_{1} \varepsilon\right), \forall t \in \mathcal{T}_{0} .
$$

These arguments are developed in Section 3 below and show that the previous theorem is a special case of the following one, which holds in infinite dimension and bounded operators $H(t, x)$.

Theorem 1.5 Assume $\mathbf{H}_{0}, \mathbf{H}_{1}$ with $\delta$ small enough, $\mathbf{H}_{2}$ and $\mathbf{H}_{3}$. Moreover, suppose that $H(t, x)$ is real, that is $\bar{H}(t, x)=H(t, x)$. Let $\omega(t)$ be the instantaneous nonlinear eigenvector defined by Proposition 1.2 in a neighbourhood $\mathcal{T}_{0}$ of $t_{0}=0$. Provided the operator $F(t)$ defined by (3.4) below is semisimple with real eigenvalues of constant multiplicity for all $t \in \mathcal{T}_{0}$, the solution $v^{\varepsilon}(t)$ to (1.2) with $v^{\varepsilon}(0)=\omega(0)$ satisfies for all $t \in \mathcal{T}_{0}$

$$
v^{\varepsilon}(t)=\mathrm{e}^{-i \int_{0}^{t} \lambda(s,[\omega(s)]) d s / \varepsilon} \omega(t)+O_{t}(\varepsilon) .
$$

As already mentioned, the assumptions of Theorem 1.3 guarantee the adequate spectral behavior of the operator $F(t)$ defined by (3.4) to get the conclusion of Theorem 1.5. In other words, assuming in $\mathbf{H}_{2}$ that all eigenvalues of the real operator $H(t, x)$ are of multiplicity one is enough to obtain the assumption on the spectral decomposition of $F(t)$. In Section 3 we describe another set of assumptions which are sufficient to satisfy the hypothesis of Theorem 1.5 in infinite dimension in the case $p=1$, see Lemma 3.1.

\subsection{Extension of the result to unbounded operators}

We now extend our results to the case where the operator $H(t, x)$ on the separable Hilbert space $\mathcal{H}$ is unbounded and takes the form $H(t, x)=H_{0}+W(t, x)$, with $W(t, x) \in \mathcal{L}(\mathcal{H})$. We make the following regularity hypothesis: 
$\mathbf{R}_{0}$ The self-adjoint operator $H_{0}$ is defined on a dense domain $\mathcal{D} \subset \mathcal{H}$, and the family of bounded operator $W(t, x)$ is self-adjoint for all $(t, x) \in \mathcal{T} \times \mathcal{X}^{p}$. Moreover, $H_{0}$, and $W(t, x)$ are real operators.

$\mathbf{R}_{1}$ The map $\mathcal{T} \times \mathcal{X}^{p} \ni(t, x) \mapsto W(t, x) \in \mathcal{L}(\mathcal{H})$ is strongly $C^{\infty}$.

$\mathbf{R}_{2}$ There exist $\delta>0$ such that $\|W(t, x)\| \leq \delta,\left\|\partial_{x_{j}} W(t, x)\right\| \leq \delta$, for all $(t, x) \in \mathcal{T} \times \mathcal{X}^{p}$ and $j \in\{1, \ldots, p\}$.

We also assume the spectral hypothesis

$\mathbf{S}_{1}$ The spectrum of $H_{0}$ consists in an infinite increasing sequence of simple eigenvalues $\lambda_{j} \geq 0, j \in \mathbb{N}$, and there exists $c_{0}>0$ and $\alpha>1 / 2$ such that the gaps satisfy

$$
\forall j \in \mathbb{N}, \quad \lambda_{j+1}-\lambda_{j} \geq c_{0} j^{\alpha} .
$$

The operator $W(t, x)$ being bounded, if $\delta$ is small enough, perturbation theory implies that for all $(t, x) \in \mathcal{T} \times \mathcal{X}^{p}$, the self-adjoint operator $H(t, x)=H_{0}+W(t, x)$ defined on $\mathcal{D}$ has spectrum $\sigma(H(t, x))=\left\{\lambda_{j}(t, x)\right\}_{j \in \mathbb{N}}$ consisting in simple eigenvalues $\lambda_{j}(t, x)$ only, and there exists $c_{1}>0$ such that the gaps satisfy for $\alpha>1 / 2$

$$
\forall(t, x) \in \mathcal{T} \times \mathcal{X}^{p}, \quad \forall j \in \mathbb{N}, \quad \lambda_{j+1}(t, x)-\lambda_{j}(t, x) \geq c_{1} j^{\alpha} .
$$

We pick some $j_{0} \in \mathbb{N}$ and assume the generic property:

$\mathbf{S}_{2}$ For all $(t, x) \in \mathcal{T} \times \mathcal{X}^{p},\left\{\lambda_{j}(t, x)-\lambda_{j_{0}}(t, x), j \in \mathbb{N}\right\} \cap\left\{-\lambda_{j}(t, x)+\lambda_{j_{0}}(t, x), j \in \mathbb{N}\right\}=\{0\}$.

Note that, since $H_{0}$ is bounded from below, this assumption concerns only a finite number of eigenvalues. Besides, this property can be inherited from a similar assumption on the eigenvalue $\lambda_{j_{0}}$ of $H_{0}$.

We consider for all $\left(t_{0}, x_{0}\right) \in \mathcal{T} \times \mathcal{X}^{p}$, the $\mathcal{C}^{\infty} \operatorname{map}(t, x) \mapsto \varphi(t, x)$ from $\mathcal{T} \times \mathcal{X}^{p}$ to $\mathcal{D} \subset \mathcal{H}$ such that

$$
H(t, x) \varphi(t, x)=\lambda_{j_{0}}(t, x) \varphi(t, x) .
$$

We drop the index $j_{0}$ as before. Provided with these properties, we can develop the same analysis as in the situation addressed above, namely, the existence of a nonlinear eigenvector and an adiabatic approximation for the nonlinear evolution equation associated with $H(t, x)$ : We consider $p$ orthonormal vectors $\left\{e_{1}, \ldots, e_{p}\right\}$ that we take in $\mathcal{D}$ for convenience, and set

$$
\forall \psi \in \mathcal{H}, \quad[\psi]=\left(\left|\left\langle e_{1} \mid \psi\right\rangle\right|^{2}, \cdots,\left|\left\langle e_{p} \mid \psi\right\rangle\right|^{2}\right) .
$$

Proposition 1.2 ensures that for any $t_{0} \in \mathcal{T}$, there exists a neighbourhood $\mathcal{T}_{0} \subset \mathcal{T}$ of $t_{0}$ such that for all $t \in \mathcal{T}_{0}$, a solution $\omega(t) \in \mathcal{D}$ of norm one to the algebraic equation (1.9) exists, see Remark 2.1. Moreover $\mathcal{T}_{0} \ni t \mapsto \omega(t)$ is $\mathcal{C}^{\infty}$ and can be chosen to satisfy $\langle\omega(t) \mid \dot{\omega}(t)\rangle \equiv 0$. Taking initial data $\omega(0)$ in $(1.2)$ gives the equation in which we are interested, namely

$$
i \varepsilon \partial_{t} \psi^{\varepsilon}(t)=\left(H_{0}+W\left(t,\left[\psi^{\varepsilon}(t)\right]\right)\right) \psi^{\varepsilon}(t), \quad \psi^{\varepsilon}(0)=\omega(0),
$$

in the weak sense on $\mathcal{D}$. By solution in the weak sense on $\mathcal{D}$ we mean the following, see [RS], vol. II, p. 284 for the linear case: For any $\chi \in \mathcal{D}$,

$$
\mathrm{i} \varepsilon \partial_{t}\left\langle\chi \mid \psi^{\varepsilon}(t)\right\rangle=\left\langle\left(H_{0}+W\left(t,\left[\psi^{\varepsilon}(t)\right]\right)\right) \chi \mid \psi^{\varepsilon}(t)\right\rangle, \quad \psi^{\varepsilon}(0)=\omega(0) .
$$


Theorem 1.6 1. Assume $\mathbf{R}_{0}$ and $\mathbf{R}_{1}$, then equation (1.14) admits a unique global solution in the weak sense of norm one.

2. Assume moreover $\mathbf{R}_{2}$ with $\delta$ small enough, $\mathbf{S}_{1}$ and $\mathbf{S}_{2}$ and let $\omega(t)$ be a $\mathcal{C}^{\infty}$ solution to (1.9) in a neighbourhood $\mathcal{T}_{0}$ of $t_{0}=0$. Then the solution $v^{\varepsilon}(t)$ to (1.2) with $v^{\varepsilon}(0)=\omega(0)$ satisfies for all $t \in \mathcal{T}_{0}$

$$
\psi^{\varepsilon}(t)=\mathrm{e}^{-\frac{i}{\varepsilon} \int_{0}^{t} \lambda(t,[\omega(t)]) d t} \omega(t)+O_{t}(\varepsilon) .
$$

The proof of Theorem 1.6 contains two things: the existence of global solutions in the weak sense, and an adiabatic approximation. The proof of the latter follows the same strategy as the one developed in Theorems 1.3 and 1.5. However, additional difficulties come from the fact that the spectrum of $F_{0}(t)$ (as defined in (1.12) consists now in an infinite sequence of eigenvalues, while one has to work in the weak topology and to be careful with domain issues when constructing Kato's operators. These points are documented in Section 4 below.

Before closing this section and discussing properties of these adiabatic solutions, we give a concrete example satisfying the assumptions of Theorem 1.6 .

Example 1.7 Consider $\mathcal{H}=L^{2}\left(\mathbb{R}_{y}\right)$ and the operator

$$
H_{0}=-\frac{1}{2} \Delta_{y}+V_{0}(y)
$$

with domain $\mathcal{D} \subset L^{2}\left(\mathbb{R}_{y}\right)$, where $V_{0}$ is a polynomial in $|y|$ with highest degree $\beta>6$. Then, as revealed by the Bohr-Sommerfeld formula $[V], H_{0}$ satisfies the assumptions $\mathbf{R}_{0}$ and $\mathbf{S}_{1}$ above. Consider $x$-dependent self-adjoint perturbations of this operator $\left(x \in \mathcal{X}^{p}\right)$

$$
H(t, x)=-\frac{1}{2} \Delta_{y}+V_{0}(y)+W(t, y, x)
$$

where $W$ is such that the map $(t, y, x) \mapsto W(t, y, x)$ is a bounded function from $\mathcal{C}^{\infty}\left(\mathcal{T} \times \mathbb{R}_{y} \times \mathcal{X}^{p}, \mathbb{R}\right)$ and there exists $\delta>0$ such that

$$
\forall(t, y, x) \in \mathcal{T} \times \mathbb{R}_{y} \times \mathcal{X}^{p}, \quad \forall j \in\{1, \ldots, p\}, \quad|W(t, y, x)|+\left|\partial_{x_{j}} W(t, y, x)\right| \leq \delta
$$

Then, $H(t, x)$ satisfies assumptions $\mathbf{R}_{1}$ and $\mathbf{R}_{2}$ above.

To be quite concrete, we can take as orthonormal basis $\left\{e_{k}\right\}_{k \in \mathbb{N}^{*}}$ of $L^{2}(\mathbb{R})$ the set of eigenstates of the harmonic oscillator, $V_{0}(y)=y^{8}, p=2$ and $W(t, y, x)=-\left(y-a(t) x_{1}\right)^{2} b(t) e^{x_{2}}$, where $a, b \in C^{\infty}(\mathbb{R}, \mathbb{R})$ with $b(t)>0$. Depending on the sign of $a(t)$, for positive values of $x_{1}, x_{2}$, the potential displays one or two wells, of various depths. The nonlinearity manifests itself by emphasising these features, depending on the amplitude of the coefficients of the solution on the first two basis vectors $e_{1}, e_{2}$, given by a Gaussian times a Hermite polynomial in $y$.

The example above is admittedly not motivated by applications to Physics, but is intended to demonstrate the adaptability of our functional framework to the unbounded setup. 


\subsection{Energy content of the solutions}

We close this introduction by discussing briefly an important feature of the solutions provided by Theorems 1.3 and 1.5. A physically relevant quantity for the nonlinear equation $(1.2)$ we consider is the instantaneous energy content of a solution $v^{\varepsilon}(t)$, defined for all $t \in \mathcal{T}_{0}$ by

$$
E_{v^{\varepsilon}}(t)=\left\langle v^{\varepsilon}(t) \mid H\left(t,\left[v^{\varepsilon}(t)\right]\right) v^{\varepsilon}(t)\right\rangle
$$

For bounded operators $\mathcal{T} \times \mathcal{X}^{p} \ni(t, x) \mapsto H(t, x) \in \mathcal{L}(\mathcal{H})$, and $\varepsilon$-independent initial conditions $v^{\varepsilon}(0)=v(0)$ the energy content satisfies the uniform bound

$$
\left|E_{v^{\varepsilon}}(t)\right| \leq \sup _{(t, x) \mathcal{T} \times \mathcal{X}^{p}}\|H(t, x)\|\|v(0)\|^{2} .
$$

For a solution of the form $v^{\varepsilon}(t)=\mathrm{e}^{-i \int_{0}^{t} \lambda(s,[\omega(s)]) d s / \varepsilon} \omega(t)+O_{t}(\varepsilon)$, the energy content simply coincides, to leading order, with the energy content of the corresponding instantaneous nonlinear eigenvalue

$$
E_{v^{\varepsilon}}(t)=\langle\omega(t) \mid H(t,[\omega(t)]) \omega(t)\rangle+O_{t}(\varepsilon)=\lambda(t,[\omega(t)])+O_{t}(\varepsilon) .
$$

In general, the behaviour in time of the energy content of a solution does not necessarily admit such a regular behaviour in the limit $\varepsilon \rightarrow 0$, which makes this property a specific feature of the adiabatic solutions.

Let us illustrate this point on the following simple example. Let $\mathbb{R} \ni t \mapsto \gamma(t) \geq \gamma_{0}>0$ and consider

$$
H(t, x)=\left(\begin{array}{cc}
0 & \gamma(t) x \\
\gamma(t) x & 0
\end{array}\right)
$$

on $\mathcal{H}=\mathbb{C}^{2}$. The evolution equation 1.2 reads

$$
i \varepsilon \partial_{t}\left(\begin{array}{l}
v_{1} \\
v_{2}
\end{array}\right)=H\left(t,\left|v_{1}\right|^{2}\right)\left(\begin{array}{l}
v_{1} \\
v_{2}
\end{array}\right)=\gamma(t)\left|v_{1}\right|^{2}\left(\begin{array}{l}
v_{2} \\
v_{1}
\end{array}\right)
$$

with initial conditions $\left(\begin{array}{l}v_{1}(0) \\ v_{2}(0)\end{array}\right)$, and the energy content of the solutions reads

$$
E_{v}(t)=\gamma(t)\left|v_{1}\right|^{2} 2 \Re\left(v_{1} \overline{v_{2}}\right)(t) .
$$

The corresponding real normalised nonlinear eigenvectors $\omega_{ \pm}(t)$ are time-independent,

$$
\omega_{ \pm}(t)=\frac{1}{\sqrt{2}}\left(\begin{array}{c}
1 \\
\pm 1
\end{array}\right)
$$

and associated to the eigenvalues $\lambda_{ \pm}\left(t,\left[\omega_{ \pm}\right]\right)= \pm \gamma(t) / 2$. Hence, the approximate solutions provided by Theorem 1.3 read

$$
v_{ \pm}(t)=e^{\mp \frac{i}{2 \varepsilon} \int_{0}^{t} \gamma(u) d u} \omega_{ \pm}(t)
$$

which turn out to be exact solutions for all $t \in \mathbb{R}$, since $\omega_{ \pm}$are time-independent. Their energy contents are thus given by

$$
E_{v_{ \pm}}(t)=E_{\omega_{ \pm}}(t)= \pm \gamma(t) / 2
$$

which is $\varepsilon$-independent. However, for general solutions $v^{\varepsilon}(t)$ the situation is different, as stated in the next Lemma which is proved in Appendix B. 
Lemma 1.8 Let $v^{\varepsilon}(t)$ be a solution of equation (1.16) with real-valued initial data such that $v_{1}(0)>0$, $v_{2}(0) \neq 0$. Then the energy content reads

$$
E_{v^{\varepsilon}}(t)=2 \gamma(t) v_{1}(0)^{3} v_{2}(0)\left[\cos ^{2}\left(\frac{\aleph(t)}{\varepsilon}\right)+\left(\frac{v_{1}(0)}{v_{2}(0)}\right)^{2} \sin ^{2}\left(\frac{\aleph(t)}{\varepsilon}\right)\right]^{-1}
$$

with $\aleph(t)=-v_{1}(0) v_{2}(0) \int_{0}^{t} \gamma(u) d u$. Hence, $E_{v^{\varepsilon}}(t) / \gamma(t)$ is actually a function of $\int_{0}^{t} \gamma(u) d u$, which oscillates between the extremal values $2 v_{1}(0)^{3} v_{2}(0)$ and $2 v_{1}(0) v_{2}(0)^{3}$ with a period of order $\varepsilon$, unless $v_{1}(0) / v_{2}(0)= \pm 1$ in which case it is a constant.

By contrast, the linear quantum adiabatic theorem implies that the energy content of any solution is given by an $\varepsilon$-independent weighted sum of instantaneous eigenvalues of the Hamiltonian, to leading order. More precisely, assume $t \mapsto H(t)$ is independent of $x$ and satisfies the hypotheses of Theorem 1.3 . Let $\left\{\varphi_{j}(t)\right\}_{j=1}^{N}$ be an orthonormal basis of instantaneous eigenvectors of $H(t)$ with phases normalised by $\left\langle\varphi_{j}(t) \mid \dot{\varphi}_{j}(t)\right\rangle \equiv 0$. Then, the energy content of $v^{\varepsilon}(t)$, solution to 1.2 , which is linear in this case, with arbitrary initial condition $v_{0}$ reads for any $t \in[0,1]$,

$$
E_{v^{\varepsilon}}(t)=\sum_{j=1}^{N}\left|\alpha_{j}\right|^{2} \lambda_{j}(t)+\mathcal{O}(\varepsilon), \quad \text { where } v_{0}=\sum_{j=1}^{N} \alpha_{j} \varphi_{j}(0) \text {. }
$$

Indeed, the linear quantum adiabatic theorem [K1] implies $v^{\varepsilon}(t)=\sum_{j=1}^{N} \alpha_{j} e^{-i \int_{0}^{t} \lambda_{j}(s) d s / \varepsilon} \varphi_{j}(t)+\mathcal{O}(\varepsilon)$, uniformly in $t \in[0,1]$, hence a direct computation of the energy content yields the above expression, thanks to $\left\langle\varphi_{j}(t) \mid \varphi_{k}(t)\right\rangle=\delta_{j, k}$.

\subsection{Organisation of the paper}

We begin by proving the existence of the instantaneous nonlinear eigenvectors in Section 2. We also discuss the limitation that may occur to their existence. This crucial part of our result is independent of the other sections and can be skipped at first reading. Then we focus in Sections 3 and 4 on the proofs of the nonlinear adiabatic Theorems to which this article is devoted to. Sections 3 deals with the case of bounded Hamiltonians, with the proofs of Theorems 1.3 and 1.5 , while we explain in Section 4 how to adapt the arguments to the unbounded setting of Theorem 1.6. Finally, two Appendices are devoted to the discussion of examples: the first one shows a situation where the spectrum of the operator $F(t)$ is not necessarily real-valued even though $H(t)$ is real, as emphasized in Remark 3.6 below; the second one focus on the analysis of the Example 1.8 .

\section{Instantaneous nonlinear eigenvectors}

We focus in this section on the existence of the generalized nonlinear eigenvector $\omega(t)$ defined in Proposition 1.2, and that we call instantaneous nonlinear eigenvectors. We first recall well-known facts in the linear setting, mainly to introduce notations. Then, we explain why a similar result remains true locally in the nonlinear regime we consider and why the obtained eigenvectors may not exist globally. 


\subsection{Existence of smooth eigenvectors in the linear adiabatic setting}

The question of local (and global) existence of $\mathcal{C}^{\infty}$ eigenvectors is simple in the linear context. Indeed, with the notations of Assumption $\mathbf{H}_{2}$ and using Riesz formula on $C_{j}(g / 2)$, a circle of radius $g / 2$ and center $\lambda_{j}(t, x)$,

$$
P_{j}(t, x)=-\frac{1}{2 \pi i} \int_{C_{j}(g / 2)}(H(t, x)-z)^{-1} d z,
$$

one gets that the projectors $P_{j}(t, x)$ 's are $\mathcal{C}^{\infty}$ as $H(t, x)$ is. Moreover, $\left\|\partial_{x_{j}} P_{j}(t, x)\right\| \leq 2 \delta / g$. The finitely degenerate eigenvalues $\lambda_{j}(t, x)=\operatorname{Tr}\left(P_{j}(t, x) H(t, x)\right)$ are thus $\mathcal{C}^{\infty}$, and the same is true if $\operatorname{Rank} P_{j}(t, x)=$ $\infty$.

Considering $j=j_{0}$, for any $\left(t_{0}, x_{0}\right) \in \mathcal{T} \times \mathcal{X}^{p}$, there exists an open neighbourhood of $\left(t_{0}, x_{0}\right)$ in which a $\mathcal{C}^{\infty}$ normalised eigenvector $\varphi_{j_{0}}(t, x) \in \mathcal{H}$ exists such that

$$
P_{j_{0}}(t, x)=\left|\varphi_{j_{0}}(t, x)\right\rangle\left\langle\varphi_{j_{0}}(t, x)\right| .
$$

Here $|\varphi\rangle\langle\psi|: \mathcal{H} \rightarrow \mathcal{H}$ maps $\eta$ to $\varphi\langle\psi \mid \eta\rangle$. More specifically, given $\varphi_{j_{0}}\left(t_{0}, x_{0}\right)$ an eigenvector of $H\left(t_{0}, x_{0}\right)$, the vector

$$
\varphi_{j_{0}}(t, x):=\frac{P_{j_{0}}(t, x) \varphi_{j_{0}}\left(t_{0}, x_{0}\right)}{\left\langle\varphi_{j_{0}}\left(t_{0}, x_{0}\right) \mid P_{j_{0}}(t, x) \varphi_{j_{0}}\left(t_{0}, x_{0}\right)\right\rangle}
$$

satisfies these conditions for all $(x, t)$ such that $P_{j_{0}}(t, x) \varphi_{j_{0}}\left(t_{0}, x_{0}\right) \neq 0$.

Actually, there exists an extension of this local map to a global $\mathcal{C}^{\infty}$ map $\mathcal{T} \times \mathcal{X}^{p} \ni(t, x) \mapsto \varphi(t, x)$, which can be viewed as follows. Using the shorthand $p=(t, x)$, set $E=\cup_{p \in \mathcal{T} \times \mathcal{X}^{p}}(p, \varphi(p))$, and $\pi: E \ni(p, \varphi(p)) \mapsto p \in \mathcal{T} \times \mathcal{X}^{p}$, so that $\pi: E \rightarrow \mathcal{T} \times \mathcal{X}^{p}$ defines a rank one vector bundle over the base $\mathcal{T} \times \mathcal{X}^{p}$. The base being contractible, it is known that the vector bundle is trivial, which is equivalent to the existence of a global $\mathcal{C}^{\infty}$ frame on the fibres of $E$, see e.g. $[\mathrm{LeP}, \mathrm{Sp}$. An alternative approach is by explicit construction, making use of the parallel transport operator defined by $(3.9)$ below. Passing to spherical coordinates $(t, x) \mapsto(r, \theta) \in \mathbb{R}^{+} \times \mathbb{S}^{p}$ and integrating the parallel transport operator along $r$, keeping $\theta$ as parameters, we get a $\mathcal{C}^{\infty}$ unit eigenvector for each $(t, x) \in \mathcal{T} \times \mathcal{X}^{p}$, by the smoothness of the eigenprojector. This property holds for $\operatorname{dim} \mathcal{H}=\infty$.

\subsection{Existence of nonlinear eigenvector}

We prove here Proposition 1.2 .

Proof: For $t_{0} \in \mathcal{T}$ fixed, dropped from the notation, $\mathbf{H}_{3}$ yields,

$$
|\varphi([\omega])\rangle\langle\varphi([\omega]) \mid \omega\rangle=\omega .
$$

This requires $\omega$ to be parallel to $\varphi([\omega])$ where the latter is normalised. We use Schauder's fixed point Theorem in a Banach space to actually prove that, locally, there exists $\omega$ such that $\omega=\varphi([\omega])$, and thus $\|\omega\|=1$. Set $B_{1}(\mathcal{H})=\{v \in \mathcal{H} \mid\|v\| \leq 1\}$ and $S: B_{1}(\mathcal{H}) \mapsto B_{1}(\mathcal{H})$ by $S(v)=\varphi([v])$. This map is well defined, continuous and $B_{1}(\mathcal{H})$ is closed, convex and nonempty. Thus $S$ will have a fixed point if $\overline{S\left(B_{1}(\mathcal{H})\right)}$ is compact. Let $K_{\varphi}=\left\{\varphi([x]) \mid x \in[0,1]^{p}\right\}$. By continuity of $\varphi$ in the variable $x$, and compactness of $[0,1]^{p}, K_{\varphi}$ is compact. Thus the closed subset $\overline{S\left(B_{1}(\mathcal{H})\right)}$ of $K_{\varphi}$ is compact and Schauder Theorem (see [E] or Theorem 2.9 in $[\mathrm{LeD}]$ ) implies the existence of a fixed point for $S$, for each given value of $t_{0}$. Since $\|\varphi([v])\| \equiv 1$, the normalization of the fixed point $\omega\left(t_{0}\right)$ holds. 
In order to prove the smoothness of the map $\mathcal{T}_{0} \ni t \mapsto \omega(t)$, we use the implicit function theorem on the $\mathcal{C}^{\infty}$ map $J: \mathcal{T} \times \mathcal{H} \times \mathcal{H} \rightarrow \mathcal{H} \times \mathcal{H}$ defined by

$$
J(t, v, \bar{v})=\left(\begin{array}{l}
J_{1}(t, v, \bar{v}) \\
J_{2}(t, v, \bar{v})
\end{array}\right)=\left(\begin{array}{c}
v-\varphi(t,[v]) \\
\bar{v}-\bar{\varphi}(t,[v])
\end{array}\right) .
$$

The zeros of $J$ define $\omega(t)$, in a neighbourhood of $\left(t_{0}, \omega\left(t_{0}\right)\right)$. Note that by a smooth change of phase we can consider locally the continuous vector $\varphi(t, x)$ defined by (2.1). For $1 \leq j \leq p$, we compute, with $\left\{e_{j}\right\}_{j \in \mathbb{N}}$ the chosen orthonormal basis of $\mathcal{H}$,

$$
\begin{array}{ll}
\partial_{v_{j}} J_{1}(t, v, \bar{v})=e_{j}-\partial_{x_{j}} \varphi(t,[v]) \bar{v}_{j}, & \partial_{\bar{v}_{j}} J_{1}(t, v, \bar{v})=-\partial_{x_{j}} \varphi(t,[v]) v_{j} \\
\partial_{v_{j}} J_{2}(t, v, \bar{v})=-\partial_{x_{j}} \bar{\varphi}(t,[v]) \bar{v}_{j}, & \partial_{\bar{v}_{j}} J_{2}(t, v, \bar{v})=e_{j}-\partial_{x_{j}} \bar{\varphi}(t,[v]) v_{j} .
\end{array}
$$

Therefore, using the notation $D_{v, \bar{v}} J(t, v, \bar{v}) \in \mathcal{L}(\mathcal{H} \times \mathcal{H})$ for the derivative with respect to the variables $(v, \bar{v}) \in \mathcal{H} \times \mathcal{H}$, we get

$$
D_{v, \bar{v}} J(t, v, \bar{v})\left(\begin{array}{l}
h \\
\bar{h}
\end{array}\right)=\left(\begin{array}{l}
h \\
\bar{h}
\end{array}\right)-\sum_{j=1}^{p}\left(\begin{array}{l}
\partial_{x_{j}} \varphi(t,[v])\left\langle v_{j} e_{j} \mid h\right\rangle+\partial_{x_{j}} \varphi(t,[v])\left\langle\bar{v}_{j} e_{j} \mid \bar{h}\right\rangle \\
\partial_{x_{j}} \bar{\varphi}(t,[v])\left\langle v_{j} e_{j} \mid h\right\rangle+\partial_{x_{j}} \bar{\varphi}(t,[v])\left\langle\bar{v}_{j} e_{j} \mid \bar{h}\right\rangle
\end{array}\right) .
$$

We recall the notation in the scalar case

$$
\partial_{z}=\frac{1}{2}\left(\partial_{x}-i \partial_{y}\right), \quad \partial_{\bar{z}}=\frac{1}{2}\left(\partial_{x}+i \partial_{y}\right)
$$

such that if $f(x, y) \equiv g(z, \bar{z})$ with $z=x+i y \in \mathbb{C}$ and $t=h+i k \in \mathbb{C}$, then

$$
D f(x, y)(h, k)=\partial_{x} f(x, y) h+\partial_{y} f(x, y) k \equiv D_{z, \bar{z}} g(z, \bar{z})(h+i k, h-i k)=\partial_{z} g(z, \bar{z}) t+\partial_{\bar{z}} g(z, \bar{z}) \bar{t} .
$$

With these notations in mind, we obtain equivalently

$$
D_{v, \bar{v}} J(t, v, \bar{v})=\operatorname{Id}-\sum_{j=1}^{p}\left|\left(\begin{array}{c}
\partial_{x_{j}} \varphi(t,[v]) \\
\partial_{x_{j}} \bar{\varphi}(t,[v])
\end{array}\right)\right\rangle\left\langle\left(\begin{array}{c}
v_{j} e_{j} \\
\bar{v}_{j} e_{j}
\end{array}\right)\right| .
$$

Therefore, for $v \in B_{1}(\mathcal{H})$, it is enough to show that $\left\|\partial_{x_{j}} \varphi(t, x)\right\|<1 / 4$, say, to satisfy the assumptions of the implicit function theorem. We compute

$$
\partial_{x_{j}} \varphi(t, x)=\frac{\partial_{x_{j}} P(t, x) \varphi\left(t_{0}, x_{0}\right)-P(t, x) \varphi\left(t_{0}, x_{0}\right)\left\langle\varphi\left(t_{0}, x_{0}\right) \mid \partial_{x_{j}} P(t, x) \varphi\left(t_{0}, x_{0}\right)\right\rangle}{\left\langle\varphi\left(t_{0}, x_{0}\right) \mid P(t, x) \varphi\left(t_{0}, x_{0}\right)\right\rangle^{2}},
$$

the norm of which is bounded above by $8 \delta / g$, in a neighbourhood of $\left(t_{0}, x_{0}\right)$ characterised by

$$
\left\|P(t, x) \varphi\left(t_{0}, x_{0}\right)\right\| \geq 2^{-1 / 4} .
$$

Hence, $\mathbf{H}_{1}$ with $\delta$ small enough yields the existence of an open neighbourhood $\mathcal{T}_{0} \ni t_{0}$ and of a $\mathcal{C}^{\infty}$ map $t \mapsto \tilde{\omega}(t)$ with $\tilde{\omega}\left(t_{0}\right)=\omega\left(t_{0}\right)$ that is solution to 1.9 for all $t \in \mathcal{T}_{0}$.

To conclude, the proof, we observe that the argument above ensures $\|\tilde{\omega}(t)\| \equiv 1$, so that the phase adjustment $\omega(t)=\tilde{\omega}(t) e^{-\int_{t_{0}}^{t}\langle\tilde{\omega}(s) \mid \dot{\tilde{\omega}}(s)\rangle d s}$ implies that $\omega(t)$ satisfies $\langle\omega(t) \mid \dot{\omega}(t)\rangle \equiv 0$.

Remark 2.1 Note that in the proof above, we have not used the assumption $H(t, x) \in \mathcal{L}(\mathcal{H})$ so that the result of Proposition 1.2 extends to unbounded families of operators $H(t, x)$. 


\section{$2.3 \quad$ Failure of global nonlinear eigenvectors}

We illustrate with an example the fact that the eigenvector constructed in Proposition 1.2 may only exists locally. For this, we consider the matrix-valued case where $H(t, x)$ is the real, symmetric, traceless two by two matrix

$$
H(t, x)=\left(\begin{array}{cc}
\cos (t \theta(x)) & \sin (t \theta(x)) \\
\sin (t \theta(x)) & -\cos (t \theta(x))
\end{array}\right),
$$

where $\mathbb{R} \ni x \mapsto \theta(x)$ is $\mathcal{C}^{\infty}$ and will be chosen later. The eigenvalues of $H(t, x)$ are +1 and -1 with associated eigenvectors

$$
V_{+}(t, x)=\left(\begin{array}{c}
\cos \left(\frac{t \theta(x)}{2}\right) \\
\sin \left(\frac{t \theta(x)}{2}\right)
\end{array}\right), \quad V_{-}(t, x)=\left(\begin{array}{c}
-\sin \left(\frac{t \theta(x)}{2}\right) \\
\cos \left(t \frac{\theta(x)}{2}\right)
\end{array}\right),
$$

respectively. We denote by $P(t, x)$ the eigenprojectors for the eigenvalue +1 . Then a real normalised vector $\omega(t)=\left(\begin{array}{l}\omega_{1}(t) \\ \omega_{2}(t)\end{array}\right)$ satisfies

$$
P(t,[\omega(t)]) \omega(t)=\omega(t)
$$

if and only if

$$
\omega_{1}(t)=\cos \left(\frac{t}{2} \theta\left(\left|\omega_{1}(t)\right|^{2}\right)\right), \omega_{2}(t)=\sin \left(\frac{t}{2} \theta\left(\left|\omega_{1}(t)\right|^{2}\right)\right)
$$

up to a global sign. It is then enough to find the function $t \mapsto \omega_{1}(t)$. For fixed $t$, it reduces to finding $Y \in[0,1]$ such that

\begin{tabular}{|c|c|c|c|c|}
\hline$y$ & 0 & $y_{\max }$ & $y_{\min }$ & 1 \\
\hline$\theta\left(y^{2}\right)$ & 0 & $\theta_{\max }$ & $\theta_{\min }$ & $\pi$ \\
\hline
\end{tabular}

$$
Y=\cos \left(\frac{t}{2} \theta\left(Y^{2}\right)\right)
$$

Let us restrict to $t \in[0,1]$ and choose the function $\theta$ according to the following picture

We fix $\theta_{\max }<\pi$ so that $\cos \frac{t}{2} \theta_{\max }>0$ for all $t \in[0,1]$ and $\cos$ is decreasing on the set of values of $\frac{t}{2} \theta$. For $t=0$, the uniqueness of the solution of the equation (2.5) is guaranteed and for $t \in(0,1]$, it depends on whether $\cos \frac{t}{2} \theta_{\max }<y_{\max }$ or not. Therefore, if we choose $y_{\max }$ and $\theta_{\max }$ such that

$$
\cos \frac{1}{2} \theta_{\max }<y_{\max }
$$

we know that there exists $\tau \in] 0,1$ [ such that the equation (2.5) has a unique solution for $t \in[0, \tau)$ and exactly three solutions for times $t \in(\tau, 1]$. Figure 1 illustrates that fact. Hence a solution chosen in a neighbourhood of $y_{\max }$ for times $t>\tau$ will disappear as $t$ passes the value $\tau$. 


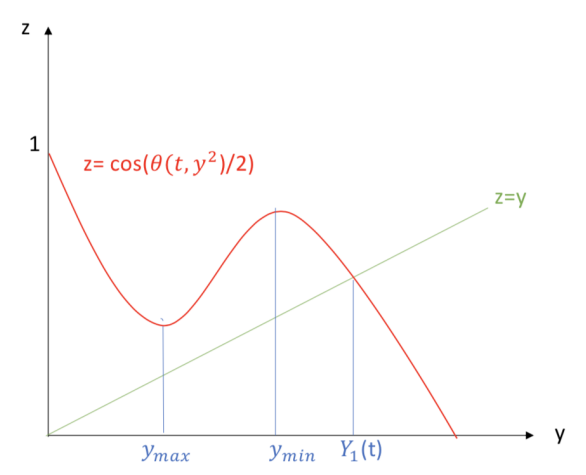

(a) $t<\tau$

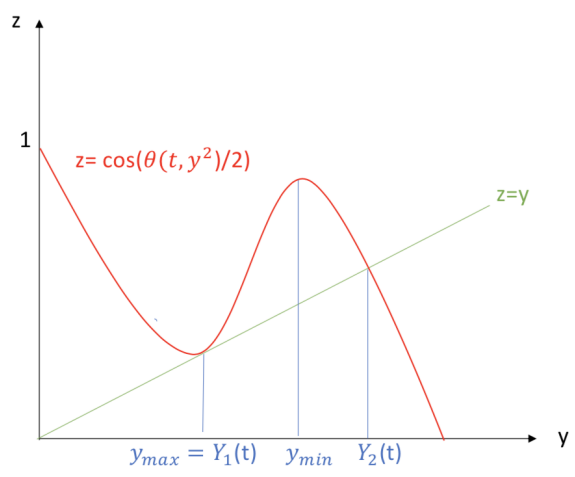

(b) $t=\tau$

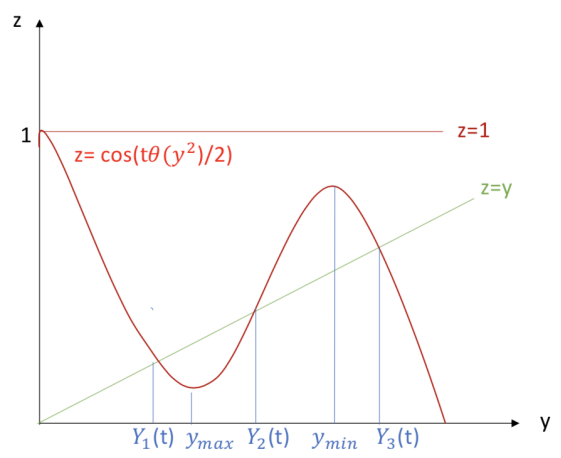

(c) $t>\tau$

Figure 1: Non uniqueness and degeneracy of the solutions of Equation 2.5: these three situations illustrating the dependance of the number of solutions depending on the value of $\cos \frac{t}{2} \theta_{\max }$ comparatively with $y_{\max }$. 


\section{The case of bounded operators}

In this section, we prove Theorems 1.3 and 1.5 the proofs of which both follow the same scheme. We first give the plan, spelling out the main steps and lemmas that we then successively prove in the next sections.

\subsection{Proof of Theorems 1.3 and 1.5}

Thanks to Lemma 1.1 with $\chi(t, x)=-\lambda(t, x)$, we can reduce the analysis to the case $\lambda(t, x)=0$ without loss of generality, by considering the shift

$$
H(t, x) \mapsto H(t, x)-\lambda(t, x) .
$$

The eigenvalues of the operator $H(t, x)$ are then all shifted by $\lambda(t, x)$ and we denote them by

$$
0 \text { and } \lambda_{j}(t, x), j \in \mathbb{N}^{*} \text {, }
$$

where the functions $\lambda_{j}(t, x)$ may have changed compared to what they were in the introduction. We set $\Delta(t)=v^{\varepsilon}(t)-\omega(t)$. Then, the map $t \mapsto \Delta(t)$ (which also depends on $\varepsilon$ ) satisfies the system

$$
i \varepsilon \dot{\Delta}(t)=H\left(t,\left[v^{\varepsilon}(t)\right]\right) v^{\varepsilon}(t)-i \varepsilon \dot{\omega}(t), \quad \Delta(0)=0,
$$

using a dot to express derivatives with respect to time. For all $t \in \mathcal{T}_{0}$, the interval $\mathcal{T}_{0}$ is the set of times around $t_{0}=0$ where $\omega(t)$ given in Proposition 1.2 exists, we have

$$
H\left(t,\left[v^{\varepsilon}(t)\right]\right)=H(t,[\omega(t)+\Delta(t)])=H(t,[\omega(t)])+2 \sum_{j=1}^{p} \partial_{x_{j}} H(t,[\omega(t)]) \Re\left(\bar{\omega}_{j} \Delta_{j}\right)+O\left(\|\Delta\|^{2}\right),
$$

and using $H(t,[\omega(t)]) \omega(t) \equiv 0$, we obtain

$$
i \varepsilon \dot{\Delta}(t)=-i \varepsilon \dot{\omega}(t)+H(t,[\omega(t)]) \Delta(t)+2 \sum_{j=1}^{p} \partial_{x_{j}} H(t,[\omega(t)]) \Re\left(\bar{\omega}_{j} \Delta_{j}\right) \omega(t)+O\left(\|\Delta\|^{2}\right) .
$$

The equation involves a source term, $-i \varepsilon \dot{\omega}(t)$, and its linear part depends on $\Delta(t)$ and $\bar{\Delta}(t)$. We write it as a system for these two vectors:

$$
\left\{\begin{array}{l}
i \varepsilon \dot{\Delta}(t)=-i \varepsilon \dot{\omega}(t)+H(t,[\omega(t)]) \Delta(t)+\sum_{j=1}^{p} \partial_{x_{j}} H(t,[\omega(t)])\left(\bar{\omega}_{j} \Delta_{j}+\omega_{j} \bar{\Delta}_{j}\right) \omega(t)+O\left(\|\Delta\|^{2}\right), \\
i \varepsilon \dot{\bar{\Delta}}(t)=-i \varepsilon \dot{\bar{\omega}}(t)-\bar{H}(t,[\omega(t)]) \bar{\Delta}(t)-\sum_{j=1}^{p} \partial_{x_{j}} \bar{H}(t,[\omega(t)])\left(\bar{\omega}_{j} \Delta_{j}+\omega_{j} \bar{\Delta}_{j}\right) \bar{\omega}(t)+O\left(\|\Delta\|^{2}\right) .
\end{array}\right.
$$

We set for $j \in\{1, \cdots, p\}$,

$$
v_{j}(t)=\partial_{x_{j}} H(t,[\omega(t)]) \omega(t),
$$

and, for later purposes, we notice that it follows from $P(t, x) H(t, x) \equiv 0$ that

$$
\left(\partial_{x_{j}} P(t, x)\right) H(t, x)+P(t, x)\left(\partial_{x_{j}} H(t, x)\right) \equiv 0
$$

and, together with $H(t,[\omega(t)]) \omega(t) \equiv 0$, we get

$$
v_{j}(t)=(\mathrm{Id}-P(t,[\omega(t)])) v_{j}(t) .
$$


We also set, for $j \in\{1, \cdots, p\}$,

$$
\mu_{j}=\left(\begin{array}{c}
v_{j} \\
-\bar{v}_{j}
\end{array}\right) \text { and } \nu_{j}=\left(\begin{array}{c}
\omega_{j} e_{j} \\
\bar{\omega}_{j} e_{j}
\end{array}\right)
$$

and rewrite the system as 1.11 , namely

$$
i \varepsilon\left(\begin{array}{c}
\dot{\Delta} \\
\dot{\Delta}
\end{array}\right)=-i \varepsilon\left(\begin{array}{c}
\dot{\omega} \\
\dot{\omega}
\end{array}\right)+F(t)\left(\begin{array}{c}
\Delta \\
\bar{\Delta}
\end{array}\right)+\left(\begin{array}{c}
r^{\varepsilon} \\
-\bar{r}^{\varepsilon}
\end{array}\right), \Delta(0)=0
$$

with $r^{\varepsilon}(t)=O\left(\|\Delta(t)\|^{2}\right)$ and

$$
F(t)=F_{0}(t)+G(t)
$$

with

$$
F_{0}(t)=\left(\begin{array}{cc}
H(t,[\omega(t)]) & 0 \\
0 & -\bar{H}(t,[\omega(t)])
\end{array}\right),
$$

and

$$
\begin{aligned}
G(t) & \left.=\sum_{j=1}^{p}\left(\begin{array}{cc}
\bar{\omega}_{j}(t)\left|v_{j}(t)\right\rangle\left\langle e_{j}\right| & \left.\omega_{j}(t) v_{j}(t)\right\rangle\left\langle e_{j}\right| \\
-\bar{\omega}_{j}(t)\left|\bar{v}_{j}(t)\right\rangle\left\langle e_{j}\right| & -\omega_{j}(t)\left|\bar{v}_{j}(t)\right\rangle\left\langle e_{j}\right|
\end{array}\right)=\sum_{j=1}^{p} \mid \begin{array}{c}
v_{j}(t) \\
-\bar{v}_{j}(t)
\end{array}\right)\left\langle\begin{array}{c}
\omega_{j}(t) e_{j} \\
\bar{\omega}_{j}(t) e_{j}
\end{array}\right| \\
& =\sum_{j=1}^{p}\left|\mu_{j}(t)\right\rangle\left\langle\nu_{j}(t)\right| .
\end{aligned}
$$

Note that $F(t), G(t) \in \mathcal{L}(\mathcal{H} \times \mathcal{H}), F(t)$ is non self-adjoint and $G(t)$ is of finite rank. Hence, $G(t)$ can be treated as a perturbation of the self-adjoint operator $F_{0}(t)$. One then observes that two classical consequences of Weinstein-Aronszajn formula are that $\sigma_{\text {ess }}(F(t))=\sigma_{\text {ess }}\left(F_{0}(t)\right)$, and that $\sigma_{d}(F(t))$ consists in finitely many of eigenvalues (see e.g. [K2], Chap. IV, §6).

The structure of the spectrum of $F(t)$ is crucial for our analysis. As we shall see in the following, the proof of Theorems 1.3 and 1.5 works out when the spectrum of $F(t)$ is semisimple with real eigenvalues of constant multiplicity for all $t \in \mathcal{T}_{0}$. Moreover, there are situations where this can be proved and the next lemma describes such cases. According to the assumptions of Theorems 1.3 and 1.5, we focus on the case where $H(t,[\omega(t)])$ is real.

Lemma 3.1 a) There exists $\delta_{0}>0$ such that if, for all $t \in \mathcal{T}_{0}$, we have $\mathbf{H}_{0}, \mathbf{H}_{1}$ for some $\delta<\delta_{0}, \mathbf{H}_{2}$ and $\mathbf{H}_{3}$, then $0 \in \sigma(F(t))$ as a doubly degenerate isolated eigenvalue, with corresponding eigennilpotent $\mathbb{N}_{0}(t) \equiv 0$.

b) Moreover, if $H(t, x)=\bar{H}(t, x), \sigma(H(t, x))$ is simple and $\sigma(H(t, x)) \cap \sigma(-H(t, x))=\{0\}$ for all $(t, x) \in \mathcal{T}_{0} \times \mathcal{X}^{p}$, then $\delta_{0}$ can be chosen so that the spectrum of $F(t)$ is real-valued for all $t \in \mathcal{T}_{0}$ and takes the form

$$
-\ell_{N-1}(t)<\cdots<-\ell_{1}(t)<0<\ell_{1}(t)<\cdots<\ell_{N-1}(t),
$$

where $\ell_{0}(t) \equiv 0$ is of multiplicity two, and each eigenvalue $\pm \ell_{k}(t), 1 \leq k \leq N-1$ is simple.

c) Finally, in the special case $p=1$, we have a) and if moreover $H(t, x)=\bar{H}(t, x)$ and $\sigma(F(t)) \backslash$ $\sigma\left(F_{0}(t)\right)$ consists in exactly $2(N-1)$ perturbed eigenvalues, then $\sigma(F(t)) \subset \mathbb{R}$ and all corresponding eigennilpotents are zero. 
Recall that the eigennilpotents correspond to the Jordan blocks in finite dimension. The points a) and b) imply that under the assumptions of Theorem 1.3 , the spectrum of $F(t)$ is semisimple with real eigenvalues of constant multiplicity for all $t \in \mathcal{T}_{0}$, and thus that the assumptions of Theorem 1.5 are satisfied. The point c) gives another situation with possibly degenerate eigenvalues where the assumptions of Theorem 1.5 hold.

Remark 3.2 i) Note that for $b)$, it is enough to assume $\sigma(H(0, x)) \cap \sigma(-H(0, x))=\{0\}$, at the cost of making $\left|\mathcal{T}_{0}\right|$ smaller. This is a generic hypothesis which automatically satisfied whenever $\lambda_{j_{0}}$ is the ground state or the upper eigenvalue.

ii) The condition \# $\left\{\sigma(F(t)) \backslash \sigma\left(F_{0}(t)\right)\right\}=2(N-1)$ states that the spectral effect of the rank one perturbation $G(t)$ is maximal, which is a genericity assumption. The multiplicities of the eigenvalues of $F_{0}(t)$ are arbitrary, possibly infinite, so that case $\left.c\right)$ does not necessary reduce to finite dimension, in contrast to the situation dealt with in Theorem 1.3 .

iii) Besides, if the spectral effect of the rank one perturbation is maximal on $\mathcal{T}_{0}$, then $\sigma(F(t))$ takes the form (3.6) for all $t \in \mathcal{T}_{0}$, with $4(N-1)$ non zero distinct eigenvalues instead of $2(N-1), 2(N-1)$ of which are simple (provided all eigenvalues of $F_{0}(t)$ have multiplicity at least two).

iv) The condition $H(t, x)$ real does not seem strong enough to ensure $\sigma(F(t)) \subset \mathbb{R}$ for $p \geq 2$; see the example of the Hamiltonian given by equation (A.1) in Appendix A.

We postpone the proof of Lemma 3.1 to Section 3.2 and we go to the next step of the proof which consists in controlling the adiabatic limit of the two-parameter evolution operator $T^{\varepsilon}(t, s)$ generated by $F(t)$ (see 3.13 below), and using it to estimate $\left\|\left(\frac{\Delta}{\Delta}\right)\right\|$ via Duhamel formula. Since $F(t)$ is not self-adjoint, this requires some care because the possible occurence of nilpotent operators in its spectral decomposition leads to subexponential divergence of the semigroup as $\varepsilon \rightarrow 0$ (see [J3]), that we cannot accommodate. However, Lemma 3.1 ensures that under the assumptions of Theorem 1.3 , and by hypothesis in Theorem 1.5, for all $t \in \mathcal{T}_{0}, F(t)$ is semi-simple, with spectral decomposition

$$
F(t)=\sum_{j=-N^{\prime}}^{N^{\prime}} \ell_{j}(t) \mathbb{P}_{j}(t) \text {, with the convention } \ell_{-|j|}(t)=-\ell_{|j|}(t)<0,
$$

where we have set $N^{\prime}=N-1$ for convenience and where $\mathbb{P}_{j}(t)$ are $\mathcal{C}^{\infty}$ eigenprojectors corresponding to real eigenvalues $\ell_{j}(t)$. We now work under these assumptions.

Despite the eigenprojectors $\mathbb{P}_{j}(t)$ not being orthogonal, with norms possibly larger than 1 , we prove in the next lemma that any operator $F(t)$ with real spectrum satisfying (3.7) generates an evolution operator which is uniformly bounded in $\varepsilon$ and almost intertwines its eigenprojectors in the adiabatic limit, in the sense of (3.10) below. In line with Kato's approach ([K1] and e.g. [HJ]), we introduce the dynamical phase operator $\Phi^{\varepsilon}(t, s)$ defined by

$$
\Phi^{\varepsilon}(t, s)=\sum_{j=-N^{\prime}}^{N^{\prime}} \mathbb{P}_{j}(0) \mathrm{e}^{-\frac{i}{\varepsilon} \int_{s}^{t} \ell_{j}(\sigma) d \sigma}, \text { s.t. } \Phi^{\varepsilon}(t, s)^{-1}=\Phi^{\varepsilon}(s, t),
$$

and the intertwining operator $W(t)$ given by

$$
i \partial_{t} W(t)=K(t) W(t), \quad W(0)=\mathrm{Id}, \text { with } K(t)=i \sum_{j=-N^{\prime}}^{N^{\prime}} \dot{\mathbb{P}}_{j}(t) \mathbb{P}_{j}(t) .
$$


As is well known (see [K2]), for all $t \in \mathbb{R}$, we have

$$
\mathbb{P}_{j}(t) W(t)=W(t) \mathbb{P}_{j}(0),
$$

and thanks to Lemma 3.1. $\left\|\Phi^{\varepsilon}(t, s)\right\|$ is uniformly bounded in $\varepsilon$. Moreover, we check that

$$
i \varepsilon \partial_{t} \Phi^{\varepsilon}(t, s)=W(t)^{-1} F(t) W(t) \Phi^{\varepsilon}(t, s) \equiv \tilde{F}(t) \Phi^{\varepsilon}(t, s) .
$$

We then introduce the bounded family of operators

$$
V^{\varepsilon}(t, s)=W(t) \Phi^{\varepsilon}(t, s) W(s)^{-1},
$$

which satisfy $V^{\varepsilon}(t, s)^{-1}=V^{\varepsilon}(s, t)$ and

$$
V^{\varepsilon}(t, s) \mathbb{P}_{j}(s)=\mathbb{P}_{j}(t) V^{\varepsilon}(t, s)=W(t) \mathbb{P}_{j}(0) \mathrm{e}^{-\frac{i}{\varepsilon} \int_{s}^{t} \ell_{j}(\sigma) d \sigma} W(s)^{-1} .
$$

Moreover, because $F(t)$ is semi-simple, $V^{\varepsilon}(t, s)$ approximates the evolution operator generated by $F(t)$, as described by the next lemma which applies in a quite general setting.

Lemma 3.3 Let $\mathcal{T}$ be an open bounded interval of $\mathbb{R}$ containing 0 and consider the operator defined on a Hilbert space $\mathcal{K}$ for all $(t, s) \in \mathcal{T} \times \mathcal{T}$ by the strong differential equation

$$
i \varepsilon \partial_{t} T^{\varepsilon}(t, s)=F(t) T^{\varepsilon}(t, s), \quad T^{\varepsilon}(s, s)=\mathrm{Id} .
$$

If $F \in C^{2}(\mathcal{T}, \mathcal{L}(\mathcal{K}))$ with continuous derivatives at $\partial \mathcal{T}$ and if $F(t)$ is semi-simple and satisfies (3.7) for all $t \in \overline{\mathcal{T}}$, then we have in $\mathcal{L}(\mathcal{K})$,

$$
T^{\varepsilon}(t, s)=V^{\varepsilon}(t, s)+O_{t, s}(\varepsilon),
$$

which implies the uniform boundedness of the family of operators $\left(T^{\varepsilon}(t, s)\right)_{\varepsilon>0}$.

Remark 3.4 i) As a consequence, $T^{\varepsilon}(t, s) \mathbb{P}_{j}(s)-\mathbb{P}_{j}(t) T^{\varepsilon}(t, s)=O_{t, s}(\varepsilon)$.

ii) Note that $N^{\prime}$ in (3.7) is independent of $t \in \mathcal{T}$, the multiplicities of the eigenvalues of $F(t)$ are arbitrary, possibly infinite.

We postpone the proof of this lemma to Section 3.3 below and conclude the proof of Theorem 1.5 . As already mentioned, Lemma 3.1 ensures we can apply Lemma 3.3 to $\mathcal{K}=\mathcal{H} \times \mathcal{H}$ and $\mathcal{T}=\mathcal{T}_{0}$ under the assumptions of Theorems 1.3 and 1.5 . We write

$$
\begin{aligned}
\left(\begin{array}{c}
\Delta(t) \\
\bar{\Delta}(t)
\end{array}\right) & =-\int_{0}^{t} T^{\varepsilon}(t, s)\left(\begin{array}{c}
\dot{\omega}(s) \\
\dot{\bar{\omega}}(s)
\end{array}\right) d s-\frac{i}{\varepsilon} \int_{0}^{t} T^{\varepsilon}(t, s)\left(\begin{array}{c}
r^{\varepsilon}(s) \\
-\bar{r}^{\varepsilon}(s)
\end{array}\right) d s \\
& =-\int_{0}^{t} V^{\varepsilon}(t, s)\left(\begin{array}{c}
\dot{\omega}(s) \\
\dot{\bar{\omega}}(s)
\end{array}\right) d s-\frac{i}{\varepsilon} \int_{0}^{t} T^{\varepsilon}(t, s)\left(\begin{array}{c}
r^{\varepsilon}(s) \\
-\bar{r}^{\varepsilon}(s)
\end{array}\right) d s+O_{t}(\varepsilon) .
\end{aligned}
$$

It follows the definition of $\omega(t)$ that $\mathbb{P}_{0}(t)\left(\begin{array}{c}\dot{\omega}(t) \\ \dot{\omega}(t)\end{array}\right)=0$ for all time $t \in \mathcal{T}_{0}$. Therefore, a classical adiabatic argument (that we spell out in Section 3.3 below) yields that Lemma 3.3 has the consequence stated below. 
Corollary 3.5 For all $t \in \mathcal{T}_{0}$, we have

$$
\int_{0}^{t} V^{\varepsilon}(t, s)\left(\begin{array}{c}
\dot{\omega}(s) \\
\dot{\bar{\omega}}(s)
\end{array}\right) d s=O_{t}(\varepsilon) .
$$

Therefore, focusing on the first component of (3.14) and setting

$$
\delta_{\tau}^{\varepsilon}=\sup _{t \in[0, \tau]}\|\Delta(t)\|
$$

with $\tau \in \mathcal{T}_{0}$, we deduce from the above that there exist $a, b>0$ such that

$$
\delta_{\tau}^{\varepsilon} \leq \varepsilon a+\frac{b}{\varepsilon} \tau \delta_{\tau}^{\varepsilon 2} .
$$

Setting $X_{\varepsilon}(\tau)=\varepsilon^{-1} \delta_{\tau}^{\varepsilon}$, we are led to study of the second order equation

$$
b \tau X^{2}-X+a \geq 0 .
$$

Since $X_{\varepsilon}(0)=0$, we deduce that $X_{\varepsilon}(\tau) \leq \frac{1}{2 b \tau}(1-\sqrt{1-4 a b \tau})=2 a /(1+\sqrt{1-4 a b \tau})$, as long as $4 a b \tau \leq 1$. Finally, we obtain

$$
\forall \tau \in\left[0,(4 a b)^{-1}\right] \cap \mathcal{T}_{0}, \quad \delta_{\tau}^{\varepsilon} \leq 2 a \varepsilon .
$$

To justify the estimate $(1.13)$ for $t$ small, we start from 3.14 to get the existence of $\alpha, \beta>0$ such that

$$
\delta_{\tau}^{\varepsilon} \leq \alpha \tau+\frac{\beta}{\varepsilon} \tau \delta_{\tau}^{\varepsilon 2}
$$

Focusing on times $\tau \leq \varepsilon$, we consider $\delta_{\tau}^{\varepsilon} \leq \alpha \tau+\beta \delta_{\tau}^{\varepsilon 2}$, which, by a similar argument using $\delta_{0}^{\varepsilon}=0$, implies, as long as $4 \alpha \beta \tau \leq 1, \delta_{\tau}^{\varepsilon} \leq 2 \alpha \tau$. Increasing the constant $\alpha$ if necessary, we get 1.13 .

The two next subsections are respectively devoted to the spectral analysis of $F(t)$ with the proof of Lemma 3.1, and to the non self-adjoint adiabatic estimates with the proof of Lemma 3.3 and its Corollary 3.5 .

\subsection{Spectral analysis of $F(t)$}

We proceed with the proof of Lemma 3.1, which relies on a careful analysis of the eigenvalues of $F(t)$ and of their multiplicity.

Recall that $C$ denotes the anti-unitary involution defined on $\mathcal{H}$ by $C \psi=\bar{\psi}$ for all $\psi \in \mathcal{H}$. It is at this stage of the proof that we shall use the assumption $H=C H C=\bar{H}$, which implies $\omega=C \omega=\bar{\omega}$ and $v_{j}=C v_{j}=\bar{v}_{j}$ for all $1 \leq j \leq p$. Due to assumption $\mathbf{H}_{1}$, we consider the operator $F(t)$ as a perturbation of the bloc diagonal operator $F_{0}(t)$. Hence, since $\sigma(\bar{H}(t,[\omega(t)]))=\sigma(H(t,[\omega(t)]))$,

$$
\sigma\left(F_{0}(t)\right)=\sigma(H(t,[\omega(t)])) \cup \sigma(-H(t,[\omega(t)])) .
$$

By our genericity assumption, and due to the reduction we have made to the case where $\lambda(t,[\omega(t)]) \equiv 0$, the spectrum of $F_{0}(t)$ consists of $2 N-1=2 N^{\prime}+1$ isolated eigenvalues

$$
-\left|\lambda_{N^{\prime}}(t,[\omega(t)])\right|<\cdots<-\left|\lambda_{1}(t,[\omega(t)])\right|<0<\left|\lambda_{1}(t,[\omega(t)])\right|<\cdots<\left|\lambda_{N^{\prime}}(t,[\omega(t)])\right| .
$$

Since the operator $G(t)$ is of small norm by assumption $\mathbf{H}_{1}$ and its definition (equations (3.2) and 3.5)), the spectrum of $F(t)$ can be inferred from that of $F_{0}(t)$ by perturbation theory. Hence $F(t)$ has eigenvalues located in small discs $\mathcal{B}_{j}^{ \pm}$centered at $\pm \lambda_{j}(t,[\omega(t)])$ and in a disk $\mathcal{B}_{0}$ with center 0 . One can assume that these disks are of same radius $r>0$ and that they do not intersect. Besides 
- in $\mathcal{B}_{j}^{ \pm}, F(t)$ has as many eigenvalues (counted with multiplicity) as the multiplicity of $\lambda_{j}(t,[\omega(t)]$ ) as an eigenvalue of $F_{0}(t)$, and in case the multiplicity is infinite, there are only finitely many eigenvalues of $F(t)$ in $\mathcal{B}_{j}^{ \pm}$that differ from $\lambda_{j}(t,[\omega(t)])$,

- in $\mathcal{B}_{0}, F(t)$ has at most two eigenvalues (counted with multiplicity).

We are going to use symmetry considerations to prove that these eigenvalues are real-valued and have the same symmetry properties as those of $F_{0}(t)$.

Remark 3.6 We develop in Appendix $A$ an argument showing that the spectrum of $F(t)$ is not necessarily real if $H(t, x)$ is real, in order to motivate the assumptions that its eigenvalues are simple.

Proof: a) We start by considering the spectrum of $F(t)$ in a neighbourhood of zero. For any $z \in \mathcal{B}_{0} \backslash\{0\}$, we can write

$$
F(t)-z=\left(F_{0}(t)-z\right)\left[\operatorname{Id}+\left(F_{0}(t)-z\right)^{-1} G(t)\right] .
$$

Introducing the spectral projector $\tilde{P}_{0}(t)$ associated with the doubly degenerate eigenvalue zero of $F_{0}(t)$ and the corresponding reduced resolvent acting on $\tilde{Q}_{0}(t)(\mathcal{H} \times \mathcal{H}), \tilde{Q}_{0}(t)=\operatorname{Id}-\tilde{P}_{0}(t)$, we have for $z \in \mathcal{B}_{0} \backslash\{0\}$,

$$
\left(F_{0}(t)-z\right)^{-1}=-\frac{\tilde{P}_{0}(t)}{z}+\left(F_{0}(t)-z\right)_{\tilde{Q}_{0}(t)}^{-1}
$$

where we denote by $A_{\tilde{Q}_{0}}$ the restriction of the operator $A$ to the range of $\tilde{Q}_{0}$. Since

$$
\tilde{P}_{0}(t)=\left(\begin{array}{cc}
|\omega(t)\rangle\langle\omega(t)| & 0 \\
0 & |\omega(t)\rangle\langle\omega(t)|
\end{array}\right)
$$

and $\left\langle\omega(t) \mid v_{j}(t)\right\rangle \equiv 0$ for all $1 \leq j \leq p$, see 3.3$)$, we get $\tilde{P}_{0}(t) G(t) \equiv 0$ so that,

$$
(F(t)-z)^{-1}=\left[\mathrm{Id}+\left(F_{0}(t)-z\right)_{\tilde{Q}_{0}(t)}^{-1} G(t)\right]^{-1}\left(F_{0}(t)-z\right)^{-1} .
$$

Indeed, the reduced resolvent is analytic in $z \in \mathcal{B}_{0}$ and $\|G(t)\|=2 \delta$, so for $\delta_{0}$ small enough, the square bracket is invertible. Therefore, the only singularity of the resolvent of $F(t)$ lies at $z=0$, which remains a doubly degenerate eigenvalue after perturbation. The corresponding spectral projector is

$$
\mathbb{P}_{0}(t)=-\frac{1}{2 i \pi} \int_{\partial \mathcal{B}_{0}}(F(t)-z)^{-1} d z=\left[\mathrm{Id}+F_{0}(t)_{\tilde{Q}_{0}(t)}^{-1} G(t)\right]^{-1} \tilde{P}_{0}(t) .
$$

and, in view of (3.17) and (3.18), the corresponding eigennilpotent $\mathbb{N}_{0}(t)=F(t) \mathbb{P}_{0}(t)$ writes, (see [K2] Chapt. III, §5)

$$
\begin{aligned}
\mathbb{N}_{0}(t) & =-\frac{1}{2 i \pi} \int_{\partial \mathcal{B}_{0}} z(F(t)-z)^{-1} d z \\
& =-\frac{1}{2 i \pi} \int_{\partial \mathcal{B}_{0}}\left[\operatorname{Id}+\left(F_{0}(t)-z\right)_{\tilde{Q}_{0}(t)}^{-1} G(t)\right]^{-1}\left[-\tilde{P}_{0}(t)+\left(F_{0}(t)-z\right)_{\tilde{Q}_{0}(t)}^{-1} z\right] d z .
\end{aligned}
$$

Since the integrand is analytic in $\mathcal{B}_{0}$, we get that $\mathbb{N}_{0}(t) \equiv 0$, which ends the proof of a) of Lemma 3.1 
b) The perturbation $G(t)$ being of finite rank, we compute the Aronszajn-Weinstein determinant ([K2], p. 245) which reads in our case for all $z \in \rho\left(F_{0}(t)\right)$, the resolvent set of $F_{0}(t)$,

$$
\begin{aligned}
w(z) & =\operatorname{det}\left(\delta_{j, k}+\left\langle\nu_{k}(t) \mid\left(F_{0}(t)-z\right)^{-1} \mu_{j}(t)\right\rangle\right)_{1 \leq j, k \leq p} \\
& =\operatorname{det}\left(\delta_{j, k}+\bar{\omega}_{k}\left\langle e_{k}(t) \mid(H(t)-z)^{-1} v_{j}(t)\right\rangle+\omega_{k}\left\langle(H(t)+\bar{z})^{-1} v_{j}(t) \mid e_{k}(t)\right\rangle\right)_{1 \leq j, k \leq p} .
\end{aligned}
$$

It follows that $w(z)=\overline{w(-\bar{z})}$ for all $z \in \mathcal{B}_{0}$. Since the zeros of $w(z)$ yield the eigenvalues of $F(t)$ in $\rho\left(F_{0}(t)\right)$, we obtain

$$
z \in \sigma(F(t)) \cap \rho\left(F_{0}(t)\right) \Leftrightarrow-\bar{z} \in \sigma(F(t)) \cap \rho\left(F_{0}(t)\right) .
$$

Since $\bar{H}(t, x)=H(t, x)$, we deduce

$$
w(z)=\operatorname{det}\left(\delta_{j, k}+\omega_{k}\left\langle e_{k}(t) \mid\left((H(t)-z)^{-1}+(H(t)+z)^{-1}\right) v_{j}(t)\right\rangle\right)_{1 \leq j, k \leq p}=w(-z) .
$$

It follows then that

$$
z \in \sigma(F(t)) \cap \rho\left(F_{0}(t)\right) \Rightarrow\{z, \bar{z},-z,-\bar{z}\} \in \sigma(F(t)) \cap \rho\left(F_{0}(t)\right) .
$$

The nonzero eigenvalues of $F_{0}(t)$ being simple by assumption, the same is true by perturbation theory for those of $F(t)$ and (3.23) shows they must be real. Moreover, these conclusions hold for any $t \in \mathcal{T}_{0}$ under the stated hypotheses.

c) We now assume $p=1$. Let $t \in \mathcal{T}_{0}$ fixed and let us drop the time variable. We make use of (1.5), with a possible relabelling of the eigenvalues due to the shift (3.1), to write with $N^{\prime}=N-1$

$$
(H-z)^{-1}=\frac{P_{0}}{-z}+\sum_{j=1}^{N^{\prime}} \frac{P_{j}}{\lambda_{j}-z}, \text { where } \lambda_{j} \neq 0 \text { if } j \geq 1 \text {, and } \lambda_{0}=0 .
$$

Thus, with $p=1, z \in \rho\left(F_{0}\right)$, and $P_{0} v_{1}=0$,

$$
w(z)=1+2 \omega_{1} \sum_{j=1}^{N^{\prime}} \frac{\lambda_{j}\left\langle e_{1} \mid P_{j} v_{1}\right\rangle}{\lambda_{j}^{2}-z^{2}}=\frac{\Pi_{k=1}^{N^{\prime}}\left(\lambda_{k}^{2}-z^{2}\right)+2 \omega_{1} \sum_{j=1}^{N^{\prime}} \prod_{\substack{k=1 \\ k \neq j}}^{N^{\prime}}\left(\lambda_{k}^{2}-z^{2}\right) \lambda_{j}\left\langle e_{1} \mid P_{j} v_{1}\right\rangle}{\Pi_{k=1}^{N^{\prime}}\left(\lambda_{k}^{2}-z^{2}\right)} .
$$

The numerator is a polynomial of degree $2 N^{\prime}$ which, by assumption, possesses $2 N^{\prime}$ distinct simple roots in $\rho\left(F_{0}\right)$. These roots being in the neighbourhood of $\sigma\left(F_{0}\right) \backslash\{0\}$ for $\delta$ small, (3.23) implies that they are real. This proves $\sigma(F) \subset \mathbb{R}$.

We now consider the eigennilpotents. The potentially nonzero eigennilpotents $\mathbb{N}_{ \pm \lambda_{j}}$ are thus attached to the unperturbed eigenvalues $\pm \lambda_{j}$ with sufficient multiplicity, i.e. with $\operatorname{dim} \tilde{P}_{j} \geq 3$ only. For $p=1$ and $z \notin \mathbb{R}$, the resolvent takes the explicit form

$$
\begin{aligned}
(F-z)^{-1} & =\left\{\operatorname{Id}-\frac{\left(F_{0}-z\right)^{-1}|\mu\rangle\langle\nu|}{1+\left\langle\nu \mid\left(F_{0}-z\right)^{-1} \mu\right\rangle}\right\}\left(F_{0}-z\right)^{-1} \\
& =\left\{\operatorname{Id}-\frac{\omega_{1}}{w(z)} \sum_{j=1}^{N^{\prime}}\left|\begin{array}{c}
\frac{P_{j} v_{1}}{\lambda_{j}-z} \\
\frac{P_{j} v_{1}}{\lambda_{j}+z}
\end{array}\right\rangle\left\langle\begin{array}{l}
e_{1} \\
e_{1}
\end{array}\right|\right\}\left(F_{0}(t)-z\right)^{-1}
\end{aligned}
$$


The eigennilpotents are the coefficients, up to a sign, of the poles of order two of the resolvent at the eigenvalues. We consider the nonzero eigenvalue $\lambda_{k}$ only, $-\lambda_{k}$ being similar. Using the fact that the numerator $\tilde{w}(z)$ of $w(z)$ in (3.24) is nonzero at $\lambda_{k}$ by assumption, we have in a neighbourhood of $\lambda_{k}$

$$
w(z)^{-1}=\left(\lambda_{k}-z\right)\left(\lambda_{k}+z\right) \Pi_{j \neq k}\left(\lambda_{j}^{2}-z^{2}\right) / \tilde{w}(z):=\left(\lambda_{k}-z\right) s_{k}\left(1+O\left(\lambda_{k}-z\right)\right),
$$

with $s_{k}=2 \lambda_{k} \Pi_{j \neq k}\left(\lambda_{j}^{2}-\lambda_{k}^{2}\right) / \tilde{w}\left(\lambda_{k}\right) \neq 0$. Hence, for $z$ close to $\lambda_{k}$,

$$
\begin{aligned}
(F-z)^{-1} & =\left\{\operatorname{Id}-\omega_{1} s_{k}\left|\begin{array}{c}
P_{k} v_{1} \\
0
\end{array}\right\rangle\left\langle\begin{array}{c}
e_{1} \\
e_{1}
\end{array}\right|+O\left(z-\lambda_{k}\right)\right\}\left(\begin{array}{cc}
\frac{P_{k}}{\lambda_{k}-z} & 0 \\
0 & 0
\end{array}\right)+O(1) \\
& =\frac{1}{\lambda_{k}-z}\left(\begin{array}{cc}
P_{k}\left(\operatorname{Id}-\omega_{1} s_{k}\left|v_{1}\right\rangle\left\langle e_{1}\right|\right) P_{k} & 0 \\
0 & 0
\end{array}\right)+O(1)
\end{aligned}
$$

The absence of pole of order two shows that $\mathbb{N}_{k}=0$, and the computation above further yields

$$
\mathbb{P}_{k}=\left(\begin{array}{cc}
P_{k}\left(\mathrm{Id}-\omega_{1} s_{k}\left|v_{1}\right\rangle\left\langle e_{1}\right|\right) P_{k} & 0 \\
0 & 0
\end{array}\right)
$$

which concludes the proof.

We end the argument by briefly checking that $\mathbb{P}_{k}$ is a projector on $\mathcal{H} \times \mathcal{H}$, or equivalently that $\left.P_{k}-\omega_{1} s_{k} P_{k}\left|v_{1}\right\rangle\left\langle e_{1}\right|\right) P_{k}$ is a projector on $\mathcal{H}$. Since $P_{k}^{2}=P_{k}$

$$
\left.\left.\left(P_{k}-\omega_{1} s_{k} P_{k}\left|v_{1}\right\rangle\left\langle e_{1}\right|\right) P_{k}\right)^{2}=P_{k}-2 \omega_{1} s_{k} P_{k}\left|v_{1}\right\rangle\left\langle e_{1}\right|\right) P_{k}+\omega_{1}^{2} s_{k}^{2} P_{k}\left|v_{1}\right\rangle\left\langle e_{1} \mid P_{k} v_{1}\right\rangle\left\langle e_{1}\right| P_{k} .
$$

The right hand side equals $\left.P_{k}-\omega_{1} s_{k} P_{k}\left|v_{1}\right\rangle\left\langle e_{1}\right|\right) P_{k}$ if $\omega_{1} s_{k}\left\langle e_{1} \mid P_{k} v_{1}\right\rangle=1$. With the definition of $s_{k}$, this is equivalent to $2 \omega_{1} \lambda_{k} \Pi_{j \neq k}\left(\lambda_{j}^{2}-\lambda_{k}^{2}\right)\left\langle e_{1} \mid P_{k} v_{1}\right\rangle=\tilde{w}\left(\lambda_{k}\right)$. Now, 3.24) gives

$$
\tilde{w}\left(\lambda_{k}\right)=\Pi_{l=1}^{N^{\prime}}\left(\lambda_{l}^{2}-\lambda_{k}^{2}\right)+2 \omega_{1} \sum_{j=1}^{N^{\prime}} \Pi_{\substack{l=1 \\ l \neq j}}^{N^{\prime}}\left(\lambda_{l}^{2}-\lambda_{k}^{2}\right) \lambda_{j}\left\langle e_{1} \mid P_{j} v_{1}\right\rangle,
$$

where the first term equals zero, while the only non zero term in the sum corresponds to $j=k$. Altogether, $\tilde{w}\left(\lambda_{k}\right)=2 \omega_{1} \Pi_{\substack{l=1 \\ l \neq k}}^{N^{\prime}}\left(\lambda_{l}^{2}-\lambda_{k}^{2}\right) \lambda_{k}\left\langle e_{1} \mid P_{k} v_{1}\right\rangle$ which yields the result.

\subsection{Non-selfadjoint adiabatic estimates}

We prove here Lemma 3.3 in a way that naturally adapts to the unbounded setting that we shall consider in Section 4 .

Proof: We first note that by the definition of $V^{\varepsilon}$ and $K$ (see $(3.12)$ and $(3.9)$ ), we have

$$
i \varepsilon \partial_{t} V^{\varepsilon}(t, s)=W(t) \sum_{j=-N^{\prime}}^{N^{\prime}} \ell_{j}(t) \mathbb{P}_{j}(0) \mathrm{e}^{-\frac{i}{\varepsilon} \int_{s}^{t} \ell_{j}\left(s^{\prime}\right) d s^{\prime}} W(s)^{-1}+\varepsilon K(t) V^{\varepsilon}(t, s) .
$$

Using (3.10) and $\mathbb{P}_{j}(0)^{2}=\mathbb{P}_{j}(0)$, we obtain

$$
\begin{aligned}
i \varepsilon \partial_{t} V^{\varepsilon}(t, s) & =\sum_{j=-N^{\prime}}^{N^{\prime}} \ell_{j}(t) \mathbb{P}_{j}(t) W(t) \mathbb{P}_{j}(0) \mathrm{e}^{-\frac{i}{\varepsilon} \int_{s}^{t} \ell_{j}\left(s^{\prime}\right) d s^{\prime}} W(s)^{-1}+\varepsilon K(t) V^{\varepsilon}(t, s) \\
& =F(t) \sum_{j=-N^{\prime}}^{N^{\prime}} \mathbb{P}_{j}(t) W(t) \mathbb{P}_{j}(0) \mathrm{e}^{-\frac{i}{\varepsilon} \int_{s}^{t} \ell_{j}\left(s^{\prime}\right) d s^{\prime}} W(s)^{-1}+\varepsilon K(t) V^{\varepsilon}(t, s),
\end{aligned}
$$


whence

$$
i \varepsilon \partial_{t} V^{\varepsilon}(t, s)=F(t) V^{\varepsilon}(t, s)+\varepsilon K(t) V^{\varepsilon}(t, s) .
$$

We can now compare $T^{\varepsilon}(t, s)$ and $V^{\varepsilon}(t, s)$. Let $\Omega^{\varepsilon}(t, s)=V^{\varepsilon}(t, s)^{-1} T^{\varepsilon}(t, s)$, we have

$$
i \partial_{t} \Omega^{\varepsilon}(t, s)=-V^{\varepsilon}(t, s)^{-1} K(t) T^{\varepsilon}(t, s)=-\left(V^{\varepsilon}(t, s)^{-1} K(t) V^{\varepsilon}(t, s)\right) \Omega^{\varepsilon}(t, s),
$$

or, equivalently

$$
\Omega^{\varepsilon}(t, s)=\mathrm{Id}+i \int_{s}^{t} V^{\varepsilon}\left(t^{\prime}, s\right)^{-1} K\left(t^{\prime}\right) V^{\varepsilon}\left(t^{\prime}, s\right) \Omega^{\varepsilon}\left(t^{\prime}, s\right) d t^{\prime} .
$$

With the shorthand $\tilde{K}\left(t^{\prime}\right)=W^{-1}\left(t^{\prime}\right) K\left(t^{\prime}\right) W\left(t^{\prime}\right)$, we have

$$
V^{\varepsilon}\left(t^{\prime}, s\right)^{-1} K\left(t^{\prime}\right) V^{\varepsilon}\left(t^{\prime}, s\right)=W(s) \Phi^{\varepsilon}\left(s, t^{\prime}\right) \tilde{K}\left(t^{\prime}\right) \Phi^{\varepsilon}\left(t^{\prime}, s\right) W^{-1}(s)
$$

and

$$
\mathbb{P}_{j}(0) \tilde{K}\left(t^{\prime}\right) \mathbb{P}_{k}(0)=i\left(1-\delta_{j, k}\right) \mathbb{P}_{j}(0) \tilde{K}\left(t^{\prime}\right) \mathbb{P}_{k}(0)
$$

Therefore, for any $j$,

$$
\mathbb{P}_{j}(s) \Omega(t, s)=\mathbb{P}_{j}(s)+i W(s) \int_{s}^{t} \mathbb{P}_{j}(0) e^{\frac{i}{\varepsilon} \int_{s}^{t^{\prime}} \ell_{j}(u) d u} \tilde{K}\left(t^{\prime}\right)\left(\operatorname{Id}-\mathbb{P}_{j}(0)\right) \Phi^{\varepsilon}\left(t^{\prime}, s\right) W^{-1}(s) \Omega^{\varepsilon}\left(t^{\prime}, s\right) d t^{\prime} .
$$

Now, observe that,

$$
i \varepsilon \partial_{t^{\prime}} e^{\frac{i}{\varepsilon} \int_{s}^{t^{\prime}} \ell_{j}(u) d u} \Phi^{\varepsilon}\left(t^{\prime}, s\right)=\tilde{F}_{j}\left(t^{\prime}\right) e^{\frac{i}{\varepsilon} \int_{s}^{t^{\prime}} \ell_{j}(u) d u} \Phi^{\varepsilon}\left(t^{\prime}, s\right)
$$

where

$$
\tilde{F}_{j}(t)=\sum_{k} \mathbb{P}_{k}(0)\left(\ell_{k}(t)-\ell_{j}(t)\right)=\tilde{F}(t)-\ell_{j}(t) \operatorname{Id}
$$

is invertible on $\left(\mathrm{Id}-\mathbb{P}_{j}(0) \mathcal{H} \times \mathcal{H}\right.$, with reduced resolvent we denote by

$$
\tilde{R}_{j}(t):=\left.\tilde{F}_{j}^{-1}(t)\right|_{\mathrm{Id}-\mathbb{P}_{j}(0)}=\sum_{\substack{k \\ k \neq j}} \mathbb{P}_{k}(0) /\left(\ell_{k}(t)-\ell_{j}(t)\right) .
$$

Thus the integrand in $(3.28)$ reads, using $(3.26)$ in the last step,

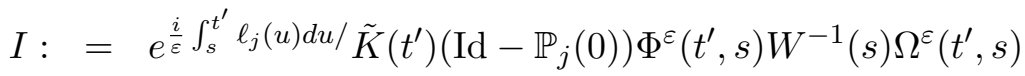

$$
\begin{aligned}
& =\tilde{K}\left(t^{\prime}\right) \tilde{R}_{j}\left(t^{\prime}\right) \tilde{F}_{j}\left(t^{\prime}\right) e^{\frac{i}{\varepsilon} \int_{s}^{t^{\prime}} \ell_{j}(u) d u} \Phi^{\varepsilon}\left(t^{\prime}, s\right) W^{-1}(s) \Omega^{\varepsilon}\left(t^{\prime}, s\right) \\
& =\tilde{K}\left(t^{\prime}\right) \tilde{R}_{j}\left(t^{\prime}\right)\left\{i \varepsilon \partial_{t^{\prime}} e^{\frac{i}{\varepsilon} \int_{s}^{t^{\prime}} \ell_{j}(u) d u} \Phi^{\varepsilon}\left(t^{\prime}, s\right)\right\} W^{-1}(s) \Omega^{\varepsilon}\left(t^{\prime}, s\right) .
\end{aligned}
$$

We deduce

$$
\begin{aligned}
I= & i \varepsilon \partial_{t^{\prime}}\left\{\tilde{K}\left(t^{\prime}\right) \tilde{R}_{j}\left(t^{\prime}\right) e^{\frac{i}{\varepsilon} \int_{s}^{t^{\prime}} \ell_{j}(u) d u} \Phi^{\varepsilon}\left(t^{\prime}, s\right) W^{-1}(s) \Omega^{\varepsilon}\left(t^{\prime}, s\right)\right\} \\
& -i \varepsilon \partial_{t^{\prime}}\left\{\tilde{K}\left(t^{\prime}\right) \tilde{R}_{j}\left(t^{\prime}\right)\right\} e^{\frac{i}{\varepsilon} \int_{s}^{t^{\prime}} \ell_{j}(u) d u} \Phi^{\varepsilon}\left(t^{\prime}, s\right) W^{-1}(s) \Omega^{\varepsilon}\left(t^{\prime}, s\right) \\
& +\varepsilon \tilde{K}\left(t^{\prime}\right) \tilde{R}_{j}\left(t^{\prime}\right) e^{\frac{i}{\varepsilon} \int_{s}^{t^{\prime}} \ell_{j}(u) d u} \tilde{K}\left(t^{\prime}\right) \Phi^{\varepsilon}\left(t^{\prime}, s\right) W^{-1}(s) \Omega^{\varepsilon}\left(t^{\prime}, s\right) .
\end{aligned}
$$


Note that thanks to our spectral hypothesis, we have

$$
\sup _{t \in \overline{\mathcal{T}}}\left\{\left\|\tilde{R}_{j}(t)\right\|,\left\|\partial_{t} \tilde{R}_{j}(t)\right\|\right\}<c_{0}
$$

for some constant $c_{0}$. We can thus integrate (3.28) by parts to get the existence of a constant $c>0$ (that may change from line to line below) such that for all $t, s \in \overline{\mathcal{T}}$

$$
\left\|\mathbb{P}_{j}(s) \Omega(t, s)-\mathbb{P}_{j}(s)\right\| \leq c c_{0} \varepsilon \mid\|\Omega\| \|,
$$

where ||$\Omega \mid\left\|=\sup _{(s, t) \in \overline{\mathcal{T}}}\right\| \Omega(t, s) \|$. Therefore,

$$
\sup _{(s, t) \in \overline{\mathcal{T}}}\|\Omega(t, s)-\mathrm{Id}\|^{2} \leq c c_{0}^{2} \varepsilon^{2}\|\| \Omega \|^{2} \leq c \varepsilon^{2}\left(\left.\||| \Omega-\mathrm{Id}\|\right|^{2}+\||| \mathrm{Id}\| \|^{2}\right),
$$

from which we get the existence of $\varepsilon_{0}>0$, independent of $t$, such that $\varepsilon<\varepsilon_{0}$ implies

$$
\||| \Omega-\operatorname{Id}|\||=O(\varepsilon) .
$$

Hence we infer the sought for bounds

$$
\Omega^{\varepsilon}(t, s)=V^{\varepsilon}(t, s)^{-1} T^{\varepsilon}(t, s)=\operatorname{Id}+O_{t, s}(\varepsilon), \text { and } T^{\varepsilon}(t, s)=O_{t, s}(1) .
$$

Let us now prove Corollary 3.5 .

Proof: Set $\chi_{\omega}(s)=\left(\begin{array}{c}\dot{\omega}(s) \\ \dot{\omega}(s)\end{array}\right)$ and recall that

$$
\tilde{P}_{0}(s) \chi_{\omega}(s)=\tilde{P}_{0}(s)\left(\begin{array}{c}
\dot{\omega}(s) \\
\dot{\bar{\omega}}(s)
\end{array}\right) \equiv 0
$$

Therefore, the perturbed projector $\mathbb{P}_{0}(s)$ associated to the kernel of $F(s)$ given by $(3.19)$ satisfies

$$
\mathbb{P}_{0}(s) \chi_{\omega}(s)=\left[\operatorname{Id}+F_{0}(s)_{\tilde{Q}_{0}(s)}^{-1} G(s)\right]^{-1} \tilde{P}_{0}(s) \chi_{\omega}(s) \equiv 0 .
$$

Hence, writing $\tilde{F}(s)=W^{-1}(s) F(s) W(s)$, we have

$$
V^{\varepsilon}(t, s) \chi_{\omega}(s)=W(t) \Phi^{\varepsilon}(t, s)\left(\operatorname{Id}-\mathbb{P}_{0}(0)\right) W^{-1}(s) \chi_{\omega}(s)=W(t) \Phi^{\varepsilon}(t, s) \tilde{F}(s)\left(\tilde{F}(s)^{-1} W^{-1}(s) \chi_{\omega}(s)\right),
$$

where $\tilde{F}(s)^{-1}$ is to be understood as the reduced resolvent of $\tilde{F}(s)$ acting on $\left(\operatorname{Id}-\mathbb{P}_{0}(0)\right) \mathcal{H} \times \mathcal{H}$. Thanks to 3.11) we can rewrite

$$
\begin{aligned}
\int_{0}^{t} V^{\varepsilon}(t, s) \chi_{\omega}(s) d s & =-\mathrm{i} \varepsilon W(t) \int_{0}^{t} \partial_{s}\left\{\Phi^{\varepsilon}(t, s)\left(\tilde{F}(s)^{-1} W^{-1}(s) \chi_{\omega}(s)\right)\right\} d s \\
& =-\left.\mathrm{i} \varepsilon W(t)\left\{\Phi^{\varepsilon}(t, s)\left(\tilde{F}(s)^{-1} W^{-1}(s) \chi_{\omega}(s)\right)\right\}\right|_{0} ^{t}=O_{t}(\varepsilon) .
\end{aligned}
$$




\section{Generalization to unbounded operators}

In this section, we prove Theorem 1.6. To start with, we focus on the existence of global weak solutions in Section 4.1. Then, to deal with the adiabatic approximation, we follow the same scheme of proof than in Section 3, analyzing the function $\Delta(t)=\psi^{\varepsilon}(t)-\omega(t)$ that solves a system of the form 1.11 but now in the weak sense (see (4.6) ). This is explained in Section 4.2. However, due to the unboundedness of the operator $H(t, x)$, several technical points have to be taken care of:

1. The existence of the propagator associated with the operator $F(t)$ (Section 4.3),

2. The analysis of the (unbounded) spectrum of $F(t)$ (Section 4.4) proving an extension of Lemma 3.1 b) with an infinite number of eigenvalues.

3. The construction of the associated adiabatic approximate propagator and of its properties (Section 4.5).

We can then conclude the proof of the Theorem 1.6 in Section 4.6 .

\subsection{Proof of Theorem $1.6(1)$}

We prove the existence of a unique global solution to the nonlinear Schrödinger equation (1.14) in the weak sense, i.e. for any $\chi \in \mathcal{D}$, we have equation (1.15), that is

$$
i \varepsilon \partial_{t}\left\langle\chi \mid \psi^{\varepsilon}(t)\right\rangle=\left\langle\left( H_{0}+W\left(t,\left[\psi^{\varepsilon}(t)\right]\right) \chi\left|\psi^{\varepsilon}(t)\right\rangle, \quad \psi^{\varepsilon}(0)=\omega(0) .\right.\right.
$$

We denote by $\mathrm{e}^{-i t H_{0}}$ the evolution group associated with $H_{0}$ which maps $\mathcal{D}$ into $\mathcal{D}$ and is differentiable on $\mathcal{D}$ only. We first consider a solution of (1.14) as an integral solution, i.e. a continuous function $t \mapsto \psi^{\varepsilon}(t) \in \mathcal{H}$ such that

$$
\forall t \in \mathcal{T}, \quad \psi^{\varepsilon}(t)=\mathrm{e}^{-\frac{i}{\varepsilon} t H_{0}} \omega(0)+\frac{1}{i \varepsilon} \int_{0}^{t} \mathrm{e}^{-\frac{i}{\varepsilon}(t-s) H_{0}} W\left(s,\left[\psi^{\varepsilon}(s)\right]\right) \psi^{\varepsilon}(s) d s .
$$

Indeed, such a $\psi^{\varepsilon}(t)$ satisfies 1.15 for all $\chi \in \mathcal{D}$. Besides, if it does exist, we will show that the solution satisfies $\left\|\psi^{\varepsilon}(t)\right\|=\|\omega(0)\|=1$.

To construct $\psi^{\varepsilon}(t)$, we consider $M \geq 1, \tau>0$ such that

$$
1+\tau M \sup \|W\| \leq M, \text { and } \sup \left(\|W\|+4 M\left\|d_{x} W\right\|\right) \tau<1,
$$

the ball $B(0, M)$ of $\mathcal{H}$ and the map $\Phi: \mathcal{C}([0, \tau \varepsilon], \mathcal{H}) \rightarrow \mathcal{C}([0, \tau \varepsilon], \mathcal{H})$

$$
\Phi: v(t) \mapsto \mathrm{e}^{-\frac{i}{\varepsilon} t H_{0}} \omega(0)+\frac{1}{i \varepsilon} \int_{0}^{t} \mathrm{e}^{-\frac{i}{\varepsilon}(t-s) H_{0}} W(s,[v(s)]) v(s) d s .
$$

By the choice of $\tau, \Phi$ maps $\mathcal{C}([0, \tau \varepsilon], B(0, M))$ into itself. Besides, $\Phi$ is a contraction:

$$
\begin{aligned}
\Phi(v)(t)-\Phi(w)(t) & =\frac{1}{i \varepsilon} \int_{0}^{t} \mathrm{e}^{-\frac{i}{\varepsilon}(t-s) H_{0}}(W(s,[v(s)]) v(s)-W(s,[w(s)]) w(s)) d s \\
& =\frac{1}{i \varepsilon} \int_{0}^{t} \mathrm{e}^{-\frac{i}{\varepsilon}(t-s) H_{0}}(W(s,[v(s)])(v(s)-w(s))-(W(s,[v(s)])-W(s,[w(s)]) w(s)) d s
\end{aligned}
$$


hence, uniformly in $t \in \overline{\mathcal{T}}$,

$$
\|\Phi(v)-\Phi(w)\| \leq \sup \left(\|W\|+4 M\left\|d_{x} W\right\|\right) \tau\|v-w\|
$$

with $\sup \left(\|W\|+4 M\left\|d_{x} W\right\|\right) \tau<1$. Therefore, $\Phi$ has a unique fixed point $\psi^{\varepsilon}(t) \in C([0, \tau \varepsilon], B(0, M))$, which is the unique integral solution of the equation 1.14 on $[0, \tau \varepsilon]$.

Now, the vector $\varphi^{\varepsilon}(t)=\mathrm{e}^{\frac{i}{\varepsilon} t H_{0}} \psi^{\varepsilon}(t)$ satisfies $\forall t \in[0, \tau \varepsilon]$,

$$
\varphi^{\varepsilon}(t)=\omega(0)+\frac{1}{i \varepsilon} \int_{0}^{t} \mathrm{e}^{\frac{i}{\varepsilon} s H_{0}} W\left(s,\left[\psi^{\varepsilon}(s)\right]\right) \mathrm{e}^{-\frac{i}{\varepsilon} s H_{0}} \varphi^{\varepsilon}(s) d s,
$$

where the integrand is continuous, so that strong differentiation with respect to time is allowed. Since the operator $\mathrm{e}^{\frac{i}{\varepsilon} t H_{0}} W\left(t,\left[\psi^{\varepsilon}(t)\right]\right) \mathrm{e}^{\frac{-i}{\varepsilon} t H_{0}}$ is self-adjoint, one gets in the usual way that,

$$
\forall t \in[0, \tau \varepsilon], \quad\left\|\varphi^{\varepsilon}(t)\right\|=\left\|\psi^{\varepsilon}(t)\right\| \equiv 1 .
$$

Observe that the choice of $\tau$ only depends on $\|W\|,\left\|d_{x} W\right\|$ and $M$, and since $\left\|\psi^{\varepsilon}(\tau \varepsilon)\right\|=1$, we can reiterate the same argument on $[\tau \varepsilon, 2 \tau \varepsilon]$ starting from the initial data $\psi^{\varepsilon}(\tau \varepsilon)$ instead of $\omega(0)$. One then constructs the unique normalised integral solution of (1.14) on $[\tau \varepsilon, 2 \tau \varepsilon]$, so that $\left\|\psi^{\varepsilon}(2 \tau \varepsilon)\right\|=1$. Iterating the process, we see that we have a unique global integral solution of the form 4.1 to the equation (1.14).

\subsection{Preparation of the proof of Theorem $1.6(2)$}

At this point, we follow the same strategy as in Section 3. Here again, the gauge invariance manifested in the conclusions of Lemma 1.1 holds in this case as well. This allows us to consider the replacement $H(t, x) \mapsto H(t, x)-\lambda(t, x) \mathrm{Id}$, keeping the notation $H(t, x)$ for the shifted Hamiltonian, which admits 0 in its spectrum and finitely many negative eigenvalues. We set $\Delta(t)=\psi^{\varepsilon}(t)-\omega(t)$, which solves a system similar to (1.11), as we now check. With the definitions

$$
\left|e_{j}\right\rangle\left\langle e_{j}\right| \omega(t)=\omega_{j}(t) e_{j}, \quad v_{j}(t)=\partial_{x_{j}} H(t,[\omega(t)]) \omega(t),
$$

and for all normalized $\chi \in \mathcal{D}$, we have, using the smoothness of the bounded operator $W(t, x)$,

$$
\begin{aligned}
i \varepsilon \partial_{t}\langle\chi \mid \Delta(t)\rangle=\left\langle\left(H_{0}+W\left(t,\left[\psi^{\varepsilon}(t)\right]\right)\right) \chi \mid \psi^{\varepsilon}(t)\right\rangle-i \varepsilon\langle\chi \mid \dot{\omega}(t)\rangle \\
=\left\langle\left(H_{0}+W(t,[\omega(t)])\right) \chi \mid \Delta\right\rangle+2 \sum_{j=1}^{p}\langle\chi|\left(\partial_{x_{j}} W(t,[\omega(t)]) \Re\left(\omega_{j} \overline{\Delta_{j}(t)}\right) \omega(t)\right\rangle \\
\quad-i \varepsilon\langle\chi \mid \dot{\omega}(t)\rangle+\left\langle\chi \mid r^{\varepsilon}(t)\right\rangle .
\end{aligned}
$$

where $r^{\varepsilon}(t)$ is of order $\|\Delta(t)\|^{2}$. Indeed, it takes the form

$$
\begin{aligned}
r^{\varepsilon}(t)= & \sum_{1 \leq j, k \leq p}\left(\Delta_{j}(t) \bar{\Delta}_{k}(t) b_{j, k}(t)+\Delta_{j}(t) \Delta_{k}(t) \tilde{b}_{j, k}(t)+\bar{\Delta}_{j}(t) \bar{\Delta}_{k}(t) \underline{b}_{j, k}(t)\right) \\
& +\sum_{1 \leq j \leq p}\left(\Delta_{j}(t) B_{j}(t) \Delta(t)+\bar{\Delta}_{j}(t) \tilde{B}_{j}(t) \Delta(t)\right.
\end{aligned}
$$


for some uniformly bounded vectors $\tilde{b}_{j, k}(t), \underline{b}_{j, k}(t), b_{j, k}(t) \in \mathcal{H}$ and uniformly bounded operators $B_{j}(t)$ and $\tilde{B}_{j}(t)$ (which may also depend on $\Delta(t)$ and $\bar{\Delta}(t)$ ):

$$
\begin{aligned}
r^{\varepsilon}(t)= & \sum_{1 \leq j \leq p} \int_{0}^{1} \partial_{x_{j}} W(t,[\omega+s \Delta])\left(2 \Re\left(\omega_{j} \bar{\Delta}_{j}\right) \Delta+\left|\Delta_{j}\right|^{2}(\Delta+\omega)\right) d s \\
& +\sum_{1 \leq j, k \leq p} \int_{0}^{1}(1-s)\left(2 \Re\left(\omega_{j} \bar{\Delta}_{j}\right)\right)\left(2 \Re\left(\omega_{k} \bar{\Delta}_{k}\right)+\left|\Delta_{k}\right|^{2}\right) d s \partial_{x_{j}, x_{k}}^{2} W(t,[\omega+s \Delta]) \omega d s .
\end{aligned}
$$

Besides, $i \varepsilon \partial_{t}\langle\chi \mid \overline{\Delta(t)}\rangle$ satisfies a similar equation corresponding to 1.11 . Thus for the nonlinear problem, we need to consider weak solutions on $\mathcal{D} \times \mathcal{D}$ of the coupled equations: For any $\left(\chi_{1}, \chi_{2}\right) \in \mathcal{D} \times \mathcal{D}$,

$i \varepsilon \partial_{t}\left\langle\left(\begin{array}{c}\chi_{1} \\ \chi_{2}\end{array}\right) \mid\left(\frac{\Delta}{\Delta}\right)\right\rangle=-i \varepsilon\left\langle\left(\begin{array}{c}\chi_{1} \\ \chi_{2}\end{array}\right) \mid\left(\begin{array}{c}\dot{\omega} \\ \dot{\omega}\end{array}\right)\right\rangle+\left\langle F^{*}(t)\left(\begin{array}{c}\chi_{1} \\ \chi_{2}\end{array}\right) \mid\left(\begin{array}{c}\Delta \\ \bar{\Delta}\end{array}\right)\right\rangle+\left\langle\left(\begin{array}{c}\chi_{1} \\ \chi_{2}\end{array}\right) \mid\left(\begin{array}{c}r^{\varepsilon}(t) \\ -\bar{r}^{\varepsilon}(t)\end{array}\right)\right\rangle, \Delta(0)=0$,

with $r^{\varepsilon}(t)=O\left(\|\Delta(t)\|^{2}\right)$ and

$$
\begin{gathered}
F(t)=F_{0}(t)+G(t) \text { with } F_{0}(t)=\left(\begin{array}{cc}
H(t,[\omega(t)]) & 0 \\
0 & -H(t,[\omega(t)])
\end{array}\right) \\
\left.G(t)=\sum_{j=1}^{p} \omega_{j}(t)\left(\begin{array}{cc}
\left|v_{j}(t)\right\rangle\left\langle e_{j}\right| & \left|v_{j}(t)\right\rangle\left\langle e_{j}\right| \\
-\left|v_{j}(t)\right\rangle\left\langle e_{j}\right| & -\left|v_{j}(t)\right\rangle\left\langle e_{j}\right|
\end{array}\right)=\sum_{j=1}^{p} \omega_{j}(t) \mid \begin{array}{c}
v_{j}(t) \\
-v_{j}(t)
\end{array}\right)\left\langle\begin{array}{c}
e_{j} \\
e_{j}
\end{array}\right| .
\end{gathered}
$$

The conjugates do not appear in the definition of $F_{0}, F$ and $G$ since assumption $\mathbf{R}_{0}$ entails the fact that $H(t, x)$ is real.

To analyse the domain of $F(t)$, it is useful to see $F_{0}(t)$ as a perturbation of $F_{0}=\left(\begin{array}{cc}H_{0} & 0 \\ 0 & -H_{0}\end{array}\right)$ by writing $F_{0}(t)=F_{0}+B(t)$ with $B(t)=\left(\begin{array}{cc}W(t,[\omega(t)]) & 0 \\ 0 & -W(t,[\omega(t)])\end{array}\right)$ bounded, self-adjoint and smooth in $t$. Indeed, this shows shows that $F_{0}(t)$ is self-adjoint on $\tilde{\mathcal{D}}:=\mathcal{D} \times \mathcal{D}$ and has domain $\tilde{\mathcal{D}}$, and the same is true for $F(t)$ since $G(t)$ is also bounded. We will also use this decomposition to analyse the existence of a two-parameter semigroup associated with $F(t)$.

In the next three paragraphs, we develop the arguments of the proof paying attention to the difficulties induced by the fact that $H_{0}$, and thus $F(t)$ are unbounded. As a fundamental preliminary, we first prove the existence of an evolution semigroup propagator associated with the operator $F(t)$. Then, the first step consists in proving that the (unbounded) spectrum of $F(t)$ consists in real eigenvalues that are all simple, except the eigenvalue zero, and the second step in constructing the associated adiabatic approximate propagator as in Lemma 3.3 and on its properties.

\subsection{Existence of a two-parameter semigroup generated by $F(t)$}

Using the latter remark, we get the following regularity result on the solutions to the linear part of the equation for $(\Delta(t), \overline{\Delta(t)})$ in $\mathcal{H} \times \mathcal{H}$. 
Lemma 4.1 Let $\mathcal{T}$ be an interval such that $0 \in \mathcal{T}$ and let $F(t)=F_{0}+B(t)+G(t)$ such that $F_{0}$ is self-adjoint on $\tilde{\mathcal{D}}=\mathcal{D} \times \mathcal{D}$ and $B(t)+G(t)$ defined for all $t \in \mathcal{T}$ is $\mathcal{C}^{\infty}$ and bounded. Then, the equation

$$
i \varepsilon \partial_{t} T^{\varepsilon}(t, s)=F(t) T^{\varepsilon}(t, s), \quad T^{\varepsilon}(s, s)=\mathrm{Id},
$$

admits a unique strong solution with values in $\tilde{\mathcal{D}}$, that is $C^{1}$ in time. Moreover, the same is true for the equation

$$
i \varepsilon \partial_{t} T^{\varepsilon}(t, s)^{*}=-T^{\varepsilon}(t, s)^{*} F^{*}(t), T^{\varepsilon}(s, s)^{*}=\mathrm{Id} .
$$

Proof: The first statement follows from Thm X.70 in [RS], see also [Kr]: the regularity assumption in time of $F(t)$ is satisfied thanks to $\mathbf{R}_{1}$ so we need to show that for all fixed $t \in \mathcal{T}_{0}, F(t)$ generates a contraction semigroup on $\mathcal{H} \times \mathcal{H}$. The operator $F_{0}(t)$ being self-adjoint on $\tilde{\mathcal{D}}$, it generates a unitary group on $\mathcal{H} \times \mathcal{H}$. Since $G(t)$ is bounded, $F(t)=F_{0}(t)+G(t)$ generates a strongly continuous semigroup $S(s)_{s \geq t}$ (see Thm III.1.3 in [EN]) which satisfies $\|S(s)\| \leq \mathrm{e}^{\|G(t)\| s}$ in the operator norm of $\mathcal{H} \times \mathcal{H}$. By rescaling, $F(t)-\|G(t)\| \mathrm{Id}$, defined on $\tilde{\mathcal{D}}$, generates a contraction semigroup, so that Thm X.70 in [RS] applies and the first statement follows.

Since the existence of a strong derivative of $T(t, s)$ on $\tilde{\mathcal{D}}$ does not imply directly the same for $T(t, s)^{*}$, we resort to the following decomposition: we write again $F(t)=F_{0}+A(t)$, where $A(t)=B(t)+G(t)$ and define the bounded operator $\Theta^{\varepsilon}$ by

$$
\Theta^{\varepsilon}(t, s)=e^{\frac{i}{\varepsilon} t F_{0}} T^{\varepsilon}(t, s) e^{-\frac{i}{\varepsilon} s F_{0}} \text {, s.t. } \Theta^{\varepsilon}(t, s)^{-1}=\Theta^{\varepsilon}(s, t)
$$

It satisfies the strong differential equation on $\mathcal{H} \times \mathcal{H}$

$$
i \varepsilon \partial_{t} \Theta^{\varepsilon}(t, s)=\tilde{A}^{\varepsilon}(t) \Theta^{\varepsilon}(t, s), \quad \Theta^{\varepsilon}(s, s)=\mathrm{Id}, \quad \text { with } \tilde{A}^{\varepsilon}(t)=e^{\frac{i}{\varepsilon} t F_{0}} A(t) e^{-\frac{i}{\varepsilon} t F_{0}} .
$$

The generator $\tilde{A}^{\varepsilon}(t)$ is strongly continuous on $\tilde{\mathcal{D}}$ and satisfies $\left\|\tilde{A}^{\varepsilon}(t)\right\|=\|A(t)\|$ for all $t \in \overline{\mathcal{T}}$. Hence we can write $\Theta^{\varepsilon}(t, s)$ as a norm convergent Dyson series, uniformly in $t \in \overline{\mathcal{T}}$, where the integrals are understood in the strong sense

$$
\Theta^{\varepsilon}(t, s)=\sum_{j \in \mathbb{N}} \Theta_{j}^{\varepsilon}(t, s), \quad \Theta_{j}^{\varepsilon}(t, s)=\left(-\frac{i}{\varepsilon}\right)^{j} \int_{s}^{t} \int_{s}^{u_{j}} \ldots \int_{s}^{u_{2}} \tilde{A}^{\varepsilon}\left(u_{j}\right) \tilde{A}^{\varepsilon}\left(u_{j-1}\right) \ldots \tilde{A}^{\varepsilon}\left(u_{1}\right) d u_{1} \ldots d u_{j-1} d u_{j} .
$$

The relation for $j \geq 1$,

$$
\Theta_{j}^{\varepsilon}(t, s)=-\frac{i}{\varepsilon} \int_{s}^{t} \tilde{A}^{\varepsilon}(u) \Theta_{j-1}^{\varepsilon}(u, s) d u
$$

allows to prove by induction that $t \mapsto \Theta_{j}^{\varepsilon}(t, s)$ is continuous in norm and, for all $\varphi \in \mathcal{H} \times \mathcal{H}$

$$
i \varepsilon \partial_{t} \Theta_{j}^{\varepsilon}(t, s) \varphi=\tilde{A}^{\varepsilon}(t) \Theta_{j-1}^{\varepsilon}(t, s) \varphi
$$

Hence $t \mapsto \Theta_{j}^{\varepsilon}(t, s)^{*}$ is norm continuous as well, and the same is true for $\Theta^{\varepsilon}(t, s)^{*}=\sum_{j \in \mathbb{N}} \Theta_{j}^{\varepsilon}(t, s)^{*}$. Moreover, $\Theta_{j}^{\varepsilon}(t, s)^{*} \psi$, for any $\psi \in \mathcal{H} \times \mathcal{H}$, satisfies for any $\varphi \in \mathcal{H} \times \mathcal{H}$

$$
\begin{aligned}
\left\langle\varphi \mid \Theta_{j}^{\varepsilon}(t, s)^{*} \psi\right\rangle & =\left\langle-\frac{i}{\varepsilon} \int_{s}^{t} \tilde{A}^{\varepsilon}(u) \Theta_{j-1}^{\varepsilon}(u, s) \varphi d u \mid \psi\right\rangle=\frac{i}{\varepsilon} \int_{s}^{t}\left\langle\tilde{A}^{\varepsilon}(u) \Theta_{j-1}^{\varepsilon}(u, s) \varphi \mid \psi\right\rangle d u \\
& =\frac{i}{\varepsilon} \int_{s}^{t}\left\langle\varphi \mid \Theta_{j-1}^{\varepsilon}(u, s)^{*} \tilde{A}^{\varepsilon}(u)^{*} \psi\right\rangle d u .
\end{aligned}
$$


Since $\tilde{A}^{\varepsilon}(t)^{*}=e^{i t F_{0} / \varepsilon} A^{*}(t) e^{-i t F_{0} / \varepsilon}$, where $t \mapsto A(t)$ is norm continuous, we get that $t \mapsto \tilde{A}^{\varepsilon}(t)^{*}$ is strongly continuous, see e.g. [ $\mathrm{Kr}]$, and so is $t \mapsto \Theta_{j-1}^{\varepsilon}(t, s)^{*} \tilde{A}^{\varepsilon}(t)^{*}$. Hence we deduce from 4 4.8] that for any $\psi \in \mathcal{H} \times \mathcal{H}$,

$$
\Theta_{j}^{\varepsilon}(t, s)^{*} \psi=\frac{i}{\varepsilon} \int_{s}^{t} \Theta_{j-1}^{\varepsilon}(u, s)^{*} \tilde{A}^{\varepsilon}(u)^{*} \psi d u,
$$

which, as above, implies for all $j \geq 1$ and all $\psi \in \mathcal{H} \times \mathcal{H}$,

$$
i \varepsilon \partial_{t} \Theta_{j}^{\varepsilon}(t, s)^{*} \psi=-\Theta_{j-1}^{\varepsilon}(t, s)^{*} \tilde{A}^{\varepsilon}(t)^{*} \psi .
$$

This differential identity allows then to get the key property

$$
i \varepsilon \partial_{t} \Theta^{\varepsilon}(t, s)^{*} \psi=-\Theta^{\varepsilon}(t, s)^{*} \tilde{A}^{\varepsilon}(t)^{*} \psi,
$$

which derives from the Dyson representation for $\Theta^{\varepsilon}(t, s)^{*}$. Therefore, $T^{\varepsilon}(t, s)^{*}=e^{-i s F_{0} / \varepsilon} \Theta^{\varepsilon}(t, s)^{*} e^{i t F_{0} / \varepsilon}$ is strongly continuously differentiable in $t$ on $\tilde{\mathcal{D}}$, since all operators in the composition are, and 4.7 . holds.

\subsection{The spectrum of $F(t)$}

We prove here that the spectrum of $F(t)$ has the required properties for $\delta$ small enough.

In that purpose, we use that, as a consequence of the hypothesis $\mathbf{S}_{2}$ :

$$
\forall(t, x) \in \mathcal{T}_{0} \times \mathcal{X}^{p}, \quad \sigma(H(t, x)) \cap \sigma(-H(t, x))=\{0\} .
$$

Note that the operator $F_{0}(t)$ satisfies the assumptions of Theorem 4.15a in [K2], with the generalization stated in b) of Remark 4.16a. We deduce that the spectrum of $F(t)$ consists in a sequence of eigenvalues

$$
\cdots<-\ell_{j}(t)<\cdots<-\ell_{2}(t)<-\ell_{1}(t)<0<\ell_{1}(t)<\ell_{2}(t)<\cdots<\ell_{j}(t)<\ldots,
$$

where $\pm \ell_{j}(t)$ are simple eigenvalues, while $\ell_{0}(t) \equiv 0$ has multiplicity 2 , with zero eigennilpotent. Each $\ell_{j}(t)$ corresponds to a unique eigenvalue of the unperturbed operator $F_{0}(t)$ determined by $H(t,[\omega(t)])$. We denote those corresponding eigenvalues of $F_{0}(t)$ by $\pm \lambda_{j}(t), j \in \mathbb{N}$ (recall that the labelling of the $\lambda_{j}$ s may differ from that of the eigenvalues of $H$. Besides, there exists a constant $c$ such that $\forall t \in \mathcal{T}_{0}, \forall j \in \mathbb{Z}$

$$
\left|\ell_{j+1}(t)-\ell_{j}(t)\right| \geq c|j|^{\alpha}, \quad \ell_{j}(t)=\ell_{j}(0)+\left(\ell_{j}(t)-\ell_{j}(0)\right), \text { and } \sup _{j \in \mathbb{Z}} \sup _{t \in \overline{\mathcal{T}_{0}}}\left|\ell_{j}(t)-\ell_{j}(0)\right|<\infty .
$$

Moreover,

$$
\forall t \in \mathcal{T}_{0}, \quad \forall j \in \mathbb{Z}, \quad\left|\dot{\ell}_{j}(t)\right| \leq c,
$$

which derives from the observation $F(t) \mathbb{P}_{j}(t)=\ell_{j}(t) \mathbb{P}_{j}(t)$ : By differentiation,

$$
\dot{A}(t) \mathbb{P}_{j}(t)+F(t) \dot{\mathbb{P}}_{j}(t)=\dot{\ell}_{j}(t) \mathbb{P}_{j}(t)+\ell_{j}(t) \dot{\mathbb{P}}_{j}(t)
$$

whence, using $\mathbb{P}_{j}(t) \dot{\mathbb{P}}_{j}(t) \mathbb{P}_{j}(t)=0$, one gets for the rank one projector $\mathbb{P}_{j}(t), j \neq 0$,

$$
\dot{\ell}_{j}(t) \mathbb{P}_{j}(t)=\mathbb{P}_{j}(t) \dot{B}(t) \mathbb{P}_{j}(t)
$$


The fact that $F(t)$ is a slightly non-selfadjoint operator in the sense of Section V.5 in [K2] allows us to apply Theorem 4.16 in [K2] and Remark 4.17 following it, to get the following spectral decomposition, under our assumption $\alpha>1 / 2$ in $\mathbf{S}_{1}$, and for $\delta_{0}$ small enough:

$$
\begin{aligned}
& F(t)=\sum_{j=-\infty}^{\infty} \ell_{j}(t) \mathbb{P}_{j}(t), \text { with the convention } \ell_{-|j|}(t)=-\ell_{|j|}(t), \text { where } \\
& \mathbb{P}_{j}(t)=\left|\Psi_{j}(t)\right\rangle\left\langle\Phi_{j}(t)\left|, j \neq 0, \quad \mathbb{P}_{0}(t)=\sum_{\sigma=1,2}\right| \Psi_{0}^{\sigma}(t)\right\rangle\left\langle\Phi_{0}^{\sigma}(t)\right|
\end{aligned}
$$

with $\left\{\Psi_{j}(t), \Phi_{j}(t)\right\}_{j \neq 0} \cup\left\{\Psi_{0}^{\sigma}(t), \Phi_{0}^{\sigma}(t)\right\}_{\sigma \in\{1,2\}}$ a biorthogonal family of vectors, with $\left\|\Psi_{j}\right\|=\left\|\Psi_{0}^{\sigma}\right\|=1$. The sum (4.11) is understood in the strong convergence sense on the time independent domain

$$
\tilde{\mathcal{D}}=\left\{\chi=\sum_{j \in \mathbb{Z}} \alpha_{j} \Psi_{j}(0) \text { s.t. } \sum_{j \in \mathbb{Z}}\left|\alpha_{j} \ell_{j}(0)\right|^{2}<\infty\right\} \subset \mathcal{H} \times \mathcal{H}
$$

Indeed, Theorem 4.16 in [K2] states that the normalised basis $\left\{\Psi_{j}(t)\right\}_{j \in \mathbb{Z}}$ is a Riesz basis, and Theorem 3.4.5 in $[\mathrm{D}$, giving a characterisation of Riesz basis, allows for the explicit description of the domain $\tilde{\mathcal{D}}$. In particular, there exist $0<C, M<\infty$ such that for all $t \in \mathcal{T}$,

$$
\begin{aligned}
& \left\|\sum_{j \in I} \mathbb{P}_{j}(t)\right\| \leq M, \quad \forall I \in \mathbb{Z} \\
& C^{-1}\|\chi\|^{2} \leq \sum_{j \in \mathbb{Z}}\left\|\mathbb{P}_{j}(t) \chi\right\|^{2} \leq C\|\chi\|^{2}, \quad \forall \chi \in \mathcal{H} \times \mathcal{H},
\end{aligned}
$$

where

$$
\begin{gathered}
\chi=\sum_{\substack{j \in \mathbb{Z} \\
j \neq 0}} \alpha_{j}(t) \Psi_{j}(t)+\sum_{\sigma=1}^{2} \Psi_{0}^{\sigma}(t) \alpha_{0}^{\sigma}(t) \\
\mathbb{P}_{j}(t) \chi=\left\langle\Phi_{j}(t) \mid \chi\right\rangle \Psi_{j}(t), \quad \forall j \neq 0 \\
\mathbb{P}_{0}(t) \chi=\sum_{\sigma=1}^{2}\left\langle\Phi_{0}^{\sigma}(t) \mid \chi\right\rangle \Psi_{0}^{\sigma}(t)
\end{gathered}
$$

Note that the domain of $H_{0}$ is

$$
\mathcal{D}=\left\{\varphi=\sum_{k \in \mathbb{N}} \beta_{k} \varphi_{k} \text { s.t. } \sum_{k \in \mathbb{N}}\left|\beta_{j} \lambda_{k}\right|^{2}<\infty\right\}
$$

where $\left(\lambda_{k}, \varphi_{k}\right)$ are the eigenvectors and eigenvalues of $H_{0}$. The reader can refer to the paper [GZ], for example, in which Riesz spectral systems are studied.

\subsection{The adiabatic propagator and its properties}

We now focus on the construction of the adiabatic propagator as in Lemma 3.3. Since its proof follows that of the bounded case, we only have to focus on domain issues. 
In view of what we have done in the previous sections, we can define, as in the bounded case, the dynamical phase operator $\Phi^{\varepsilon}(t, s)$ (see $(3.8)$ and $\left.(3.9)\right)$

$$
\Phi^{\varepsilon}(t, s)=\sum_{j=-\infty}^{\infty} \mathbb{P}_{j}(0) \mathrm{e}^{-\frac{i}{\varepsilon} \int_{s}^{t} \ell_{j}(\sigma) d \sigma}, \text { s.t. } \Phi^{\varepsilon}(t, s)^{-1}=\Phi^{\varepsilon}(s, t),
$$

which is a family of uniformly bounded operators that map $\tilde{\mathcal{D}}$ on $\tilde{\mathcal{D}}$, thanks to 4.15. At this point, further making use of (4.9) and of the fact that $\left|\left(e^{i x}-1\right) / x\right|$ is uniformly bounded in $x \in \mathbb{R}$, one sees by a dominated convergence argument that $t \mapsto \Phi^{\varepsilon}(t, s)$ is also a strongly continuously differentiable two-parameter evolution operator on $\tilde{\mathcal{D}}$, where 3.11 holds.

We also define the intertwining operator $W(t)$ given by

$$
i \partial_{t} W(t)=K(t) W(t), \quad W(0)=\mathrm{Id}, \text { with } K(t)=i \sum_{j=-\infty}^{\infty} \dot{\mathbb{P}}_{j}(t) \mathbb{P}_{j}(t)
$$

It is shown in Proposition 3.1 and Lemma 3.2 of [J3], that as soon as $\alpha>0, K(t)$ is well defined, $\mathcal{C}^{\infty}$, and $W(t)$ satisfies the intertwining property (3.10) with each of the projectors.

Actually, theses properties of $W$ are shown in [J] for orthogonal projectors $\mathbb{P}_{j}(t)$. However, as a routine inspection reveals, the proofs hold mutatis mutandis in the non selfadjoint case, provided the growing gap assumption $\mathbf{S}$ holds, and the resolvent $(F(t)-z)^{-1}$ can be bounded in an approximate way by the inverse of the distance to the spectrum. Our perturbative framework, characterised by $\delta_{0}$ small ensures that this is the case.

We then introduce the bounded family of operators

$$
V^{\varepsilon}(t, s)=W(t) \Phi^{\varepsilon}(t, s) W(s)^{-1},
$$

which map $\tilde{\mathcal{D}}$ on $\tilde{\mathcal{D}}$ and satisfy $V^{\varepsilon}(t, s)^{-1}=V^{\varepsilon}(s, t)$, together with

$$
V^{\varepsilon}(t, s) \mathbb{P}_{j}(s)=\mathbb{P}_{j}(t) V^{\varepsilon}(t, s)=W(t) \mathbb{P}_{j}(0) \mathrm{e}^{-\frac{i}{\varepsilon} \int_{s}^{t} \ell_{j}(\sigma) d \sigma} W(s)^{-1} .
$$

The latter intertwining property implies that $W(t)$ maps $\tilde{\mathcal{D}}$ on $\tilde{\mathcal{D}}$ : From 4.11 and the definition of $\Phi_{j}(t)$ and $\Psi_{j}(t)$ in 4.12 , for $j \neq 0$,

$$
W(t)\left|\Psi_{j}(0)\right\rangle\left\langle\Phi_{j}(0)|=| \Psi_{j}(t)\right\rangle\left\langle\Phi_{j}(t)\right| W(t) \Rightarrow W(t) \Psi_{j}(0)=\Psi_{j}(t)\left\langle\Phi_{j}(t) \mid W(t) \Psi_{j}(0)\right\rangle,
$$

so that we have the following property: if $\chi \in \tilde{D}$, see 4.13$)$, with coefficient $\alpha_{j}=\left\langle\Phi_{j}(0) \mid \chi\right\rangle, j \neq 0$, in the basis at time 0 , then $W(t) \chi$ has an expansion in the basis at time $t$ with coefficients $\alpha_{j}(t)=$ $\left\langle\Phi_{j}(t) \mid W(t) \Psi_{j}(0)\right\rangle \alpha_{j}, j \neq 0$, where $\left|\left\langle\Phi_{j}(t) \mid W(t) \Psi_{j}(0)\right\rangle\right|$ is uniformly bounded in $j \neq 0$, thanks to (4.14).

We now describe the adjustments requested to argue as in Section 3.3 to prove the analogue of Lemma 3.3 , that is

$$
T^{\varepsilon}(t, s)=V^{\varepsilon}(t, s)+O_{t, s}(\varepsilon) .
$$

We recall that the differential equation 3.13 has to be understood in the strong sense on $\tilde{\mathcal{D}}$, and $T^{\varepsilon}(t, s)$ is $\mathcal{C}^{1}$ on $\tilde{\mathcal{D}}$ and maps $\tilde{\mathcal{D}}$ on $\tilde{\mathcal{D}}$, according to Lemma 4.1. Analogously, $V^{\varepsilon}(t, s)$ satisfies 3.25 in the strong sense on $\tilde{D}$, and the same holds for $\Omega^{\varepsilon}(t, s)$ defined by $(3.26)$. Then, integration by parts on 
the integrand of $(3.26)$ is to be understood in the strong sense, on vectors of $\tilde{D}$. To deal with 3.28 , one notes that 3.29 holds in the strong sense on $\tilde{\mathcal{D}}$, with $\tilde{F}_{i}(t)=\tilde{F}(t)-\ell_{j}(t)$ Id the closed operator on $\tilde{D}$ obtained by extending the summation to $k \in \mathbb{Z}$ in 3.30 . Similarly, its reduced resolvent on $\left(\operatorname{Id}-\mathbb{P}_{j}(0)\right) \mathcal{H} \times \mathcal{H}$ simply reads $\tilde{R}_{j}(t)=\sum_{\substack{k \in \mathbb{Z} \\ k \neq j}} \mathbb{P}_{k}(0) /\left(\ell_{k}(t)-\ell_{j}(t)\right)$. Note that thanks to 4.15 and the spectral behaviours 4.9 and 4.10 , we have with the notation $\langle j\rangle=\left(1+j^{2}\right)^{1 / 2}$

$$
\max \left\{\left\|\tilde{R}_{j}(t)\right\|,\left\|\partial_{t} \tilde{R}_{j}(t)\right\|\right\} \leq c\langle j\rangle^{-\alpha},
$$

for some constant $c$ uniform in $t \in \overline{\mathcal{T}}$, that may change from line to line below. Using this estimate in the integration by parts formula (3.31) we now get

$$
\left\|\mathbb{P}_{j}(s) \Omega(t, s)-\mathbb{P}_{j}(s)\right\| \leq c \varepsilon\langle j\rangle^{-\alpha}|\|\Omega \mid\|,
$$

where $\left|\|\Omega \mid\|=\sup _{(s, t) \in \overline{\mathcal{T}}}\|\Omega(t, s)\|\right.$. Therefore, since $2 \alpha>1$,

$$
\sup _{(s, t) \in \overline{\mathcal{T}}}\|\Omega(t, s)-\mathrm{Id}\|^{2} \leq c \varepsilon^{2}\|\| \Omega \|^{2},
$$

from which we get, as in Section 3.3 , that for $\varepsilon<\varepsilon_{0}, \varepsilon_{0}$ independent of $t$,

$$
\|\Omega-\operatorname{Id}|\||=O(\varepsilon) .
$$

In turn, this proves Lemma 3.3 in our current unbounded context.

Given the observations above, we also note that the arguments used in proof of Corollary 3.5 are valid in the unbounded case as well.

\subsection{Conclusion of the proof of Theorem 1.6 (2)}

We set

$$
\delta_{\tau}^{\varepsilon}=\sup _{t \in[0, \tau]} \sup _{\chi \in \mathcal{D},\|\chi\|=1}|\langle\chi \mid \Delta(t)\rangle|=\sup _{t \in[0, \tau]}\|\Delta(t)\| .
$$

In particular, since $e_{j} \in \mathcal{D}$ for all $j \in\{1, \cdots, p\}$, we have

$$
\forall t \in[0, \tau],\left|\Delta_{j}(t)\right| \leq \delta_{\tau}^{\varepsilon} .
$$

Besides, for any family of bounded operators $C(t)$ on $\mathcal{H} \times \mathcal{H}$, for $\left(\begin{array}{l}\chi_{1} \\ \chi_{2}\end{array}\right) \in \tilde{\mathcal{D}}$ normalized and for $0 \leq s \leq t \leq \tau$, using $(3.3)$

$$
\left|\left\langle T^{\varepsilon}(t, s)^{*}\left(\begin{array}{c}
\chi_{1} \\
\chi_{2}
\end{array}\right) \mid C(s)\left(\begin{array}{c}
\Delta(t) \\
\bar{\Delta}(t)
\end{array}\right)\right\rangle\right| \leq \sup _{s \in[0, \tau]}\left\|T^{\varepsilon}(t, s) C(s)\right\| \sqrt{2} \delta_{\tau}^{\varepsilon} \equiv \theta \sup _{s \in[0, \tau]}\|C(s)\| \delta_{\tau}^{\varepsilon} .
$$

We then deduce from 4.5 that there exists a constant $b>0$ such that for any $\left(\begin{array}{l}\chi_{1} \\ \chi_{2}\end{array}\right) \in \tilde{\mathcal{D}}$ and $0 \leq s \leq t \leq \tau$,

$$
\left|\left\langle\chi_{1} \mid r^{\varepsilon}(s)\right\rangle\right|+\left|\left\langle\chi_{2} \mid \bar{r}^{\varepsilon}(s)\right\rangle\right| \leq b\left(\delta_{\tau}^{\varepsilon}\right)^{2} .
$$


We observe that $T^{\varepsilon}(s, t)^{*}=\left(T^{\varepsilon}(t, s)^{-1}\right)^{*}$ satisfies in the strong sense on $\tilde{\mathcal{D}}$

$$
i \varepsilon \partial_{t}\left(T^{\varepsilon}(s, t)^{*}\right)=F(t)^{*} T^{\varepsilon}(s, t)^{*}, T^{\varepsilon}(s, s)^{*}=\mathrm{Id} .
$$

In view of (4.6), for any $\chi_{1}, \chi_{2} \in \mathcal{D}$,

$$
\begin{aligned}
& i \varepsilon \partial_{t}\left\langle T^{\varepsilon}(s, t)^{*}\left(\begin{array}{c}
\chi_{1} \\
\chi_{2}
\end{array}\right) \mid\left(\begin{array}{c}
\Delta(t) \\
\bar{\Delta}(t)
\end{array}\right)\right\rangle=-\left\langle F(t)^{*} T^{\varepsilon}(s, t)^{*}\left(\begin{array}{c}
\chi_{1} \\
\chi_{2}
\end{array}\right) \mid\left(\begin{array}{c}
\Delta(t) \\
\bar{\Delta}(t)
\end{array}\right)\right\rangle \\
& \quad+\left\langle F(t)^{*} T^{\varepsilon}(s, t)^{*}\left(\begin{array}{c}
\chi_{1} \\
\chi_{2}
\end{array}\right) \mid\left(\begin{array}{c}
\Delta(t) \\
\bar{\Delta}(t)
\end{array}\right)\right\rangle+\left\langle T^{\varepsilon}(s, t)^{*}\left(\begin{array}{c}
\chi_{1} \\
\chi_{2}
\end{array}\right) \mid\left(\begin{array}{c}
r^{\varepsilon}(t) \\
-\bar{r}^{\varepsilon}(t)
\end{array}\right)\right\rangle-i \varepsilon\left\langle T^{\varepsilon}(s, t)^{*}\left(\begin{array}{c}
\chi_{1} \\
\chi_{2}
\end{array}\right) \mid\left(\begin{array}{c}
\dot{\omega}(t) \\
\dot{\omega}(t)
\end{array}\right)\right\rangle,
\end{aligned}
$$

where the first term of the right hand side comes from the equation of $T^{\varepsilon}(s, t)^{*}$, and the second term comes from the fact that $\Delta$ satisfies the equation in the weak sense, making use of $T^{\varepsilon}(s, t)^{*}: \widetilde{\mathcal{D}} \rightarrow \widetilde{\mathcal{D}}$. Therefore, integrating between 0 and $s$, we obtain

$$
\left\langle\left(\begin{array}{l}
\chi_{1} \\
\chi_{2}
\end{array}\right) \mid\left(\begin{array}{c}
\Delta(s) \\
\bar{\Delta}(s)
\end{array}\right)\right\rangle=\frac{1}{i \varepsilon} \int_{0}^{s}\left\langle T^{\varepsilon}(s, t)^{*}\left(\begin{array}{c}
\chi_{1} \\
\chi_{2}
\end{array}\right) \mid\left(\begin{array}{c}
r^{\varepsilon}(t) \\
-\bar{r}^{\varepsilon}(t)
\end{array}\right)\right\rangle d t-\int_{0}^{s}\left\langle T^{\varepsilon}(s, t)^{*}\left(\begin{array}{l}
\chi_{1} \\
\chi_{2}
\end{array}\right) \mid\left(\begin{array}{c}
\dot{\omega}(t) \\
\dot{\omega}(t)
\end{array}\right)\right\rangle d t .
$$

Since $T^{\varepsilon}(s, t)^{*}\left(\begin{array}{l}\chi_{1} \\ \chi_{2}\end{array}\right) \in \mathcal{D} \times \mathcal{D}$, we can use estimate 4.20 for normalised $\left(\begin{array}{l}\chi_{1} \\ \chi_{2}\end{array}\right)$ and there exists $\widetilde{b}$ such that

$$
\left|\int_{0}^{s}\left\langle T^{\varepsilon}(s, t)^{*}\left(\begin{array}{c}
\chi_{1} \\
\chi_{2}
\end{array}\right) \mid\left(\begin{array}{c}
r^{\varepsilon}(t) \\
-\bar{r}^{\varepsilon}(t)
\end{array}\right)\right\rangle d t\right| \leq \tilde{b}|s|\left(\delta_{\varepsilon}^{s}\right)^{2} .
$$

Besides,

$$
\begin{aligned}
\int_{0}^{s}\left\langle T^{\varepsilon}(s, t)^{*}\left(\begin{array}{l}
\chi_{1} \\
\chi_{2}
\end{array}\right) \mid\left(\begin{array}{c}
\dot{\omega}(t) \\
\dot{\omega}(t)
\end{array}\right)\right\rangle d t & =\int_{0}^{s}\left\langle\left(\begin{array}{c}
\chi_{1} \\
\chi_{2}
\end{array}\right) \mid T^{\varepsilon}(s, t)\left(\begin{array}{c}
\dot{\omega}(t) \\
\dot{\omega}(t)
\end{array}\right)\right\rangle d t \\
& =\int_{0}^{s}\left\langle\left(\begin{array}{c}
\chi_{1} \\
\chi_{2}
\end{array}\right) \mid V^{\varepsilon}(s, t)\left(\begin{array}{c}
\dot{\omega}(t) \\
\dot{\omega}(t)
\end{array}\right)\right\rangle d t+O_{s}(\varepsilon)=O_{s}(\varepsilon)
\end{aligned}
$$

by Lemma 3.3 and 3.5 . Finally, by choosing $\chi_{1}=\chi, \chi_{2}=0$, we obtain that there exists constants $a, \widetilde{b}>0$, uniform in $0 \leq s \leq \tau$

$$
|\langle\chi, \Delta(s)\rangle| \leq a \varepsilon+\frac{\tilde{b}}{\varepsilon}|s|\left(\delta_{\varepsilon}^{s}\right)^{2},
$$

whence

$$
\delta_{\varepsilon}^{\tau} \leq a \varepsilon+\frac{b}{\varepsilon} \tau\left(\delta_{\varepsilon}^{\tau}\right)^{2}
$$

which allows to conclude the proof.

\section{A Appendix A}

According to Remark 3.6, we provide here an argument showing the spectrum of $F(t)$ is not necessarily real if $H(t, x)$ is real. We consider a smooth Hamiltonian $\mathbb{R} \times \mathbb{R}^{2} \ni(t, x) \mapsto H(t, x)$ on a Hilbert space $\mathcal{H}$, and of the form

$$
H(t, x)=\lambda_{1}(t, x) P_{1}(t, x)+\lambda_{2}(t, x) P_{2}(t, x) \text { with } \sum_{j=1}^{3} P_{j}(t, x) \equiv \mathrm{Id},
$$


with the assumption that the eigenvalue 0 is simple and that the $\lambda_{j}(t, x)$ are of arbitrary multiplicities $(j=1,2)$. With the assumptions of Section 3.2 , that means $N^{\prime}=p=2$ and, dropping the arguments $(t,[\omega(t)])$ in the variables, the Aronszajn-Weinstein determinant 3.21 takes the form

$$
w(z)=\operatorname{det}\left(\delta_{j k}+\sum_{l=1}^{2} \frac{2 \lambda_{l} \omega_{j}\left\langle e_{j} \mid P_{l} v_{k}\right\rangle}{\left(\lambda_{l}-z\right)\left(\lambda_{l}+z\right)}\right)_{1 \leq j, k \leq 2} .
$$

Introducing $q_{12}(z)=\left(\lambda_{1}-z\right)\left(\lambda_{1}+z\right)\left(\lambda_{2}-z\right)\left(\lambda_{2}+z\right)$ and $q_{j}(z)=\left(\lambda_{j}-z\right)\left(\lambda_{j}+z\right), j=1,2$, we have

$$
w(z)=\frac{1}{q_{12}(z)} \operatorname{det}\left(q_{12}(z) \operatorname{Id}+2\left(\begin{array}{cc}
\omega_{1}\left\langle e_{1} \mid\left(\sum_{l=1}^{2} P_{l} \lambda_{l} q_{\bar{l}}(z)\right) v_{1}\right\rangle & \omega_{1}\left\langle e_{1} \mid\left(\sum_{l=1}^{2} P_{l} \lambda_{l} q_{\bar{l}}(z)\right) v_{2}\right\rangle \\
\omega_{2}\left\langle e_{2} \mid\left(\sum_{l=1}^{2} P_{l} \lambda_{l} q_{\bar{l}}(z)\right) v_{1}\right\rangle & \omega_{2}\left\langle e_{2} \mid\left(\sum_{l=1}^{2} P_{l} \lambda_{l} q_{\bar{l}}(z)\right) v_{2}\right\rangle
\end{array}\right)\right),
$$

where $\overline{1}=2$ and $\overline{2}=1$. By assumption, all matrix elements are real-valued. If $z_{0} \in \mathbb{R} \backslash\left\{\lambda_{1}, \lambda_{2}\right\}$ is a zero of $w(z)$, that is a real eigenvalue of $F$, that means $-q_{12}\left(z_{0}\right) / 2$ is a real nonzero eigenvalue of the matrix

$$
b\left(z_{0}\right)=\left(\begin{array}{ll}
\omega_{1}\left\langle e_{1} \mid\left(\sum_{l=1}^{2} P_{l} \lambda_{l} q_{\bar{l}}\left(z_{0}\right)\right) v_{1}\right\rangle & \omega_{1}\left\langle e_{1} \mid\left(\sum_{l=1}^{2} P_{l} \lambda_{l} q_{\bar{l}}\left(z_{0}\right)\right) v_{2}\right\rangle \\
\omega_{2}\left\langle e_{2} \mid\left(\sum_{l=1}^{2} P_{l} \lambda_{l} q_{\bar{l}}\left(z_{0}\right)\right) v_{1}\right\rangle & \omega_{2}\left\langle e_{2} \mid\left(\sum_{l=1}^{2} P_{l} \lambda_{l} q_{\bar{l}}\left(z_{0}\right)\right) v_{2}\right\rangle
\end{array}\right) \in M_{2}(\mathbb{R}) .
$$

This requires $\left(\operatorname{Tr} b\left(z_{0}\right)\right)^{2}-4 \operatorname{det} b\left(z_{0}\right)>0$, which is not granted for a generic matrix in $M_{2}(\mathbb{R})$. While $b\left(z_{0}\right)$ is not completely arbitrary, it doesn't necessarily possess the symmetries that enforce this, as we argue below. Hence, the existence of nonzero real eigenvalues for $F$ cannot be inferred from the sole requirement that $H$ is real.

To be more quantitative, assume the eigenvalue $\lambda_{2}$ of $H(t, x)$ is independent of $(t, x)$. Thus $\omega(t)$ is independent of $\lambda_{2}$ that we will consider as a large parameter. Consider $t$ fixed and $z_{0}$ in the vicinity of $\lambda_{1}(t,[\omega(t)])$, assumed to be of order one. Then, for $\lambda_{2}>0$ large, we have $q_{12}\left(z_{0}\right)=\lambda_{2}^{2}\left(\lambda_{1}^{2}-z_{0}^{2}\right)+O(1)$, $q_{2}\left(z_{0}\right)=\lambda_{2}^{2}+O(1), q_{1}\left(z_{0}\right)=O(1)$ so that $\sum_{l=1}^{2} P_{l} \lambda_{l} q_{\bar{l}}\left(z_{0}\right)=P_{1} \lambda_{1} \lambda_{2}^{2}+O\left(\lambda_{2}\right)$ and

$$
b\left(z_{0}\right)=\lambda_{1} \lambda_{2}^{2}\left(\begin{array}{ll}
\omega_{1}\left\langle e_{1} \mid P_{1} v_{1}\right\rangle & \omega_{1}\left\langle e_{1} \mid P_{1} v_{2}\right\rangle \\
\omega_{2}\left\langle e_{2} \mid P_{1} v_{1}\right\rangle & \omega_{2}\left\langle e_{2} \mid P_{1} v_{2}\right\rangle
\end{array}\right)+O\left(\lambda_{2}\right) .
$$

The condition $\left(\operatorname{Tr} b\left(z_{0}\right)\right)^{2}-4 \operatorname{det} b\left(z_{0}\right)>0$ for $\lambda_{2}$ large, is equivalent to saying the $z_{0}$ independent leading order matrix in A.2 has real eigenvalues, i.e. to having

$$
\left.\left(\omega_{1}\left\langle e_{1} \mid P_{1} v_{1}\right\rangle-\omega_{2}\left\langle e_{2} \mid P_{1} v_{2}\right\rangle\right)^{2}+4 \omega_{1} \omega_{2}\left\langle e_{1} \mid P_{1} v_{2}\right\rangle\left\langle e_{2} \mid P_{1} v_{1}\right\rangle\right)>0 .
$$

Recall that given $\left(\omega_{1}, \omega_{2}\right)=[\omega]$, the operators $H([\omega]), P_{1}([\omega])$ and $\partial_{x_{j}} H([\omega]), j=1,2$ are fixed, as is $\omega=\varphi([\omega])$. Hence, the same is true for

$$
u_{j}=P_{1}([\omega]) v_{j}=P_{1}([\omega]) \partial_{x_{j}} H([\omega]) \omega \equiv K_{j}([\omega]) \omega, \text { with }\left\langle u_{j} \mid \omega\right\rangle=0, \quad j=1,2,
$$

so that $\mathrm{A} .3$ reads

$$
\left.\left(\omega_{1}\left\langle e_{1} \mid u_{1}\right\rangle-\omega_{2}\left\langle e_{2} \mid u_{2}\right\rangle\right)^{2}+4 \omega_{1} \omega_{2}\left\langle e_{1} \mid u_{2}\right\rangle\left\langle e_{2} \mid u_{1}\right\rangle\right)>0 .
$$

For generic vectors $\left\{e_{1}, e_{2}, \omega, u_{1}, u_{2}\right\}$ satisfying (A.4), the above condition needs not be true. Actually, for any real unitary operator $R$ such that $R \omega=\omega$, we have $\omega_{j}=\left\langle\omega \mid e_{j}\right\rangle=\left\langle\omega \mid R e_{j}\right\rangle$, so that $\left\{f_{1}, f_{2}\right\}=$ $\left\{R e_{1}, R e_{2}\right\}$ forms another orthonormal family defining the nonlinearity of the problem, keeping $\omega_{j}$, $j=1,2$ fixed. It can be shown that if A.5 holds for $\left\{e_{1}, e_{2}, \omega, u_{1}, u_{2}\right\}$, with $\operatorname{dim}(\mathbb{C} \omega)^{\perp} \geq 3, \omega_{j} \neq 0$, and $0<\left|\left\langle u_{1} \mid u_{2}\right\rangle\right|<\left\|u_{1}\right\|\left\|u_{2}\right\|$, a real unitary $R$ leaving $\omega$ invariant can be chosen to that (A.5) is false for $\left\{f_{1}, f_{2}, \omega, u_{1}, u_{2}\right\}$. The idea consists in discussing the restriction of $R$ to $(\mathbb{C} \omega)^{\perp}$ so that the orthonormal vectors $\left\{f_{1}, f_{2}\right\}$ have scalar products with $\left\{u_{1}, u_{2}\right\}$ which make A.5 false. 


\section{B Appendix B}

Let us look for more general solutions to $(1.16)$ and prove Lemma 1.8. Reparametrising the time variable $t \mapsto s(t)=\int_{0}^{t} \gamma(u) d u$ and writing $w(s(t))=v(t)$ allows us to get rid of the factor $\gamma(t)$,

$$
i \varepsilon \partial_{s}\left(\begin{array}{l}
w_{1} \\
w_{2}
\end{array}\right)=\left|w_{1}\right|^{2}\left(\begin{array}{l}
w_{2} \\
w_{1}
\end{array}\right) .
$$

Writing out $w_{1}(s)=x(s)+i y(s), w_{2}(s)=z(s)+i t(s)$, we get the equivalent system

$$
\left\{\begin{array}{l}
\varepsilon \dot{x}=\left(x^{2}+y^{2}\right) t \\
\varepsilon \dot{y}=-\left(x^{2}+y^{2}\right) z \\
\varepsilon \dot{z}=\left(x^{2}+y^{2}\right) y \\
\varepsilon \dot{t}=-\left(x^{2}+y^{2}\right) x
\end{array}\right.
$$

It is readily checked that the three following expressions are constants of the motion

$$
x^{2}+t^{2}, y^{2}+z^{2}, x z+y t
$$

so that the system can be solved by quadratures. Refraining from spelling out the solution in full generality, we consider solutions corresponding to the initial conditions

$$
y(0)=t(0)=0, \quad x(0)>0, z(0) \neq 0 .
$$

We get for all $s \in \mathbb{R}$ with $\alpha_{\varepsilon}(s)=-x(0) z(0) s / \varepsilon$

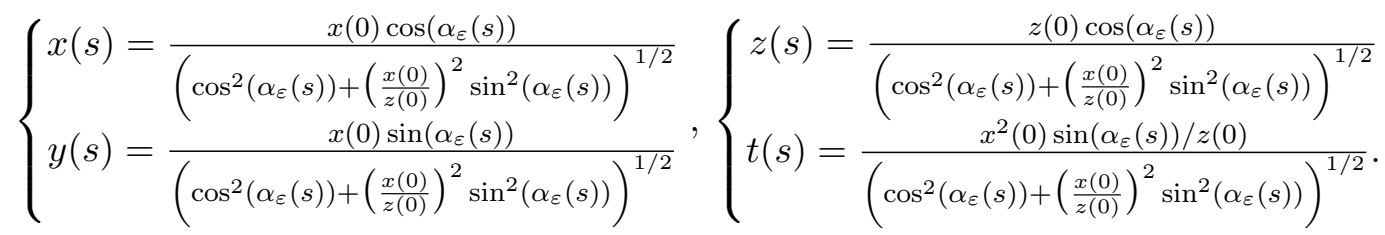

In case $x(0)=1= \pm z(0)$, we recover (1.17), modulo the reparametrization of the time variable. In all other cases, noting that $\Re\left(w_{1} \overline{w_{2}}\right)$ is conserved, we compute in the $s$ variable

$$
E_{w}(s)=2 \frac{x(0)^{3} z(0)}{\cos ^{2}\left(\alpha_{\varepsilon}(s)\right)+\left(\frac{x(0)}{z(0)}\right)^{2} \sin ^{2}\left(\alpha_{\varepsilon}(s)\right)},
$$

which gives the result of the Lemma 1.8 with $\aleph(t)=\varepsilon \alpha_{\varepsilon}(s), x(0)=v_{1}(0)$ and $z(0)=v_{2}(0)$.

\section{References}

[A-SF] Abou Salem, W., Fröhlich, J., Adiabatic theorems and reversible isothermal processes. Lett. Math. Phys. 72 (2005), p. 153-163.

[A] Agrawal, G.P., Applications of Nonlinear Fiber Optics; Academic, SanDiego (2001)

[AE] Avron, J.E., Elgart, A., Adiabatic theorem without a gap condition, Commun. Math. Phys., 203 (1999), p. 445-463. 
[AFGG] Avron, J.E., Fraas, M., Graf, G.M., Grech, P., Adiabatic theorems for generators of contracting evolutions, Commun. Math. Phys., 314 (2012), p. 163-191.

[AHS] Avron, J. E., Howland, J. S., Simon, B., Adiabatic theorems for dense point spectra, Commun. Math. Phys., 128 (1990), p. 497-507.

[ASY] Avron, J.E., Seiler, R., Yaffe, L.G., Adiabatic theorems and applications to the quantum Hall effect, Commun. Math. Phys., 110(1987), p. 33-49.

[BDR] Bachmann, S., De Roeck, W., Fraas, M., The adiabatic theorem and linear response theory for extended quantum systems, Commun. Math. Phys., 361(2018), p. 997-1027.

[BQ] Biao, W. and Qian, N., Nonlinear Landau-Zener tunneling, Phys. Rev. A, 61 (2000), p. 023402.

[BF] Born, M., Fock, V., Beweis des Adiabatensatzes. Z. Phys., 51 (1928), p. 165-180.

[CFK1] Carles, R. and Fermanian-Kammerer, C., A nonlinear adiabatic theorem for coherent states, Nonlinearity, 24 (2011) p. 1-22.

[CFK2] Carles, R. and Fermanian-Kammerer, C., A Nonlinear Landau-Zener formula, J. Stat. Phys., 152 (2012), p. 619-656.

[D] Davies, E. B., Linear operators and their spectra, Cambridge studies in advanced mathematics 106 , CUP, 2007.

[DKS] Dranov, A., Kellendonk, J. and Seiler, R., Discrete time adiabatic theorems for quantum mechanical systems, J. Math. Phys., 39 (1998), p. 1340-1349.

[E] Evans, L.C., Partial Differential Equations, Graduate Studies in Mathematics 10, American Mathematical Society, Providence, RI, 1998.

[EN] Engel, K.-J., Nagel, R., One-parameter semigroups for linear evolution equations, Springer, 2000.

[GG] Gang, Z. and Grech, P., Adiabatic theorem for the Gross-Pitaevskii equation, Commun. in PDE, 42 (2017), p. 731-756.

[GZ] Guo, B. Z., Zwart, H. J. Riesz spectral systems, Memorandum, Department of Applied Mathematics, University of Twente No. 1594 ISSN 0169-2690 (2001).

[HJ] Joye, A. and Hagedorn, G., Recent Results on Non-Adiabatic Transitions in Quantum Mechanics, AMS Contemporary Mathematics Series, 412 (2006) p. 183-198.

[HJPR1] Hanson, E. P., Joye, A., Pautrat, Y., Raquépas, R., Landauer's Principle in Repeated Interaction Systems Commun. Math. Phys., 349 (2017), p. 285-327.

[HJPR2] Hanson, E. P., Joye, A., Pautrat, Y., Raquépas, R., Landauer's Principle for Trajectories of Repeated Interaction Systems, Ann. H. Poincaré, 19 (2018), p. 1939-1991.

[H] Hari, L., Coherent States for Systems of $L^{2}$-supercritical Nonlinear Schrödinger Equations, Comm. Partial Differential Equations, 38, p. 529-573, (2013).

[Je] Jensen, S.M., The nonlinear coherent coupler, IEEE J. Quantum Electron. 18, 1580-1583, (1982) 
[J1] Joye, A., Proof of the Landau-Zener formula, Asymp. Analysis, 9 (1994), p. 209-258.

[J2] Joye, A., Absence of Absolutely Continuous Spectrum of Floquet Operators, J. Stat. Phys., 75 (1994), p. 929-952.

[J3] Joye, A., General Adiabatic Evolution with a Gap Condition, Commun. Math. Phys., 275 (2007), p. $139-162$.

[JKP] Joye, A., Kunz, H., Pfister, C.-E, Exponential Decay and Geometric Aspect of Transition Probabilities in the Adiabatic Limit, Ann. Phys., 208 (1991), p. 299-332.

[JP] Joye, A., Pfister, C.-E., Exponentially Small Adiabatic Invariant for the Schrödinger Equation, Commun. Math. Phys. 140, (1991), p. 15-41.

[K1] Kato, T., On the Adiabatic Theorem of Quantum Mechanics, J. Phys. Soc. Japan, 5 (1950), p. $435-439$.

[K2] Kato, T., Perturbation Theory for Linear Operators (Springer-Verlag Berlin Heidelberg New York 1980).

[Kh] Khomeriki, R, Nonlinear Landau-Zener tunneling in coupled waveguide arrays, Phys. Rev. A, 82 (2010), p. 013839.

[KhRu] Khomeriki, R. and Ruffo, S., Nonadiabatic Landau-Zener tunneling in waveguide arrays with a step in the refractive index, Phys. Rev. Lett., 94 (2005), p. 113904.

[Kr] Krein, S.G., Linear Differential Equations in Banach Space, Translations of Mathematical Monographs, vol. 29, AMS, 1971.

[LeD] Le Dret, H., Nonlinear Elliptic Partial Differential Equations: An Introduction, Universitext, Springer, 253 p., 2018.

[LeP] Le Potier, J., Lectures on Vector Bundles, CUP, 1997.

[J-L et al.] Jona-Lasinio, M., Morsch, O., Cristiani, M., Malossi, N., Müller, J. H., Courtade, E., Anderlini, M. and Arimondo, E., Asymmetric Landau-Zener tunneling in a periodic potential, Phys. Rev. Lett., 91 (2003), p. 230406.

[LLFY] Liu, J., Li, S.-C., Fu, L.-B., Ye,D.-F., Nonlinear Adiabatic Evolution of Quantum Systems Springer Singapore, 2018.

[MCWW] Milburn, G.J., Corney, J., Wright, E.M., Walls, D.F., Quantum dynamics of an atomic Bose-Einstein condensate in a double-well potential, Phys. Rev. A 55, (1997), p. 4318-4324.

[N1] Nenciu, G., On the adiabatic theorem of quantum mechanics, J. Phys. A, Math. Gen., 13 (1980), p. $15-18$.

[N2] Nenciu, G., Linear adiabatic theory. Exponential estimates, Commun. Math. Phys., 152 (1993), p. 479-496.

[RS] Reed, M., Simon, B., Methods of Modern Mathematical Physics, Academic Press, 1972. 
[Sc] Schmid, J., Adiabatic theorems with and without spectral gap condition for non- semisimple spectral values. In: Exner, P., König, W., Neidhardt, H. (eds.) Mathematical Results in Quantum Mechanics: Proceedings of the QMath12 Conference. World Scientific Publishing, Singapore, 2014.

[Sp] Spanier, E., Algebraic Topology, Springer, 1994.

[S] Sparber, C., Weakly Nonlinear Time-Adiabatic Theory, Ann. H. Poincaré, 17 (2016), p. 913-936.

[Ta] Tanaka, A., Adiabatic theorem for discrete time evolution, J. Phys. Soc. Japan, 80 (2011), p. 12.

[Te] Teufel, S., A note on the adiabatic theorem without gap condition, Lett. Math. Phys., 58 (2001), p. 261-266.

[V] Voros, A., Exact resolution method for general 1D polynomial Schrödinger equations, J. Phys. A 32 (1999), p.5993-6007. 